Revue des patrimoines

$37 \mid 2018$

Jardins collectifs : de l'abbé Lemire aux jardins d'insertion. Typologies - Expériences - Enjeux de conservation

\title{
Le musée municipal de l'Hygiène et sa genèse administrative. Contribution à une histoire des musées techniques municipaux à Paris
}

The creation of a municipal hygiene museum, a contribution to the history of municipal technical museums in Paris

\section{Miriam Simon}

\section{(2) OpenEdition}

\section{Journals}

Édition électronique

URL : http://journals.openedition.org/insitu/19746

DOI : 10.4000/insitu. 19746

ISSN : 1630-7305

Éditeur

Ministère de la culture

Référence électronique

Miriam Simon, « Le musée municipal de l'Hygiène et sa genèse administrative. Contribution à une histoire des musées techniques municipaux à Paris », In Situ [En ligne], 37 | 2018, mis en ligne le 20 décembre 2018, consulté le 20 avril 2019. URL : http://journals.openedition.org/insitu/19746 ; DOI : 10.4000/insitu. 19746

Ce document a été généré automatiquement le 20 avril 2019.

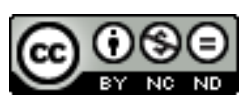

In Situ Revues des patrimoines est mis à disposition selon les termes de la licence Creative Commons Attribution - Pas d'Utilisation Commerciale - Pas de Modification 4.0 International. 


\section{Le musée municipal de l'Hygiène et sa genèse administrative. Contribution à une histoire des musées techniques municipaux à Paris}

The creation of a municipal hygiene museum, a contribution to the history of municipal technical museums in Paris

\section{Miriam Simon}

Cet article se veut une étape dans la recherche et appelle des prolongements. Il s'inscrit dans le cadre de la Mission patrimoine professionnel instituée en octobre 2015. Conscients que de nouveaux modes de travail liés à une dématérialisation croissante, la réorganisation de l'administration et de nombreux déménagements peuvent altérer le sens que les agents donnent à leur travail, le maire adjoint aux Ressources humaines et le secrétariat général de la Ville de Paris souhaitent recueillir des éléments matériels et immatériels $\mathrm{du}$ patrimoine professionnel municipal en associant les dimensions « historique, identitaire, culturelle et touristique » d'un tel projet (lettre de mission du 17 septembre 2015). Cette commande, innovante au sein de l'administration parisienne il s'agit d'articuler patrimoine matériel (vêtements professionnels, outils et objets utilisés ou fabriqués par les agents municipaux) et patrimoine immatériel (constitution d'archives audiovisuelles par la collecte de témoignages d'agents, le filmage de savoirfaire et de visites commentées), en procédant à une contextualisation de l'ensemble par des recherches documentaires et archivistiques - requiert de chercher les prémices permettant d'inscrire cette démarche dans l'histoire municipale. Le fruit de ce travail prend la forme d'une histoire administrative, en raison du périmètre des recherches effectuées en vue de la rédaction de cet article qui a pour ambition de constituer un jalon pour des recherches futures. Il s'appuie sur les archives conservées aux Archives de Paris, sur les ouvrages (annuaires administratifs, catalogues des expositions spéciales de la Ville 
de Paris aux Expositions universelles) et rapports du conseil municipal consultés à la bibliothèque de l'Hôtel de Ville, des coupures de presse et documents conservés par la Bibliothèque historique de la Ville de Paris (BHVP), ainsi que sur des lectures concernant l'histoire des égouts de Paris et celle des Expositions universelles. Il tente de reconstituer la genèse de la production d'objets d'intérêt désormais historique subsistant dans plusieurs services actuels de la Ville de Paris ${ }^{1}$.

2 Désireuse de mettre en valeur et de faire connaître l'excellence technologique et la capacité d'innovation de ses services sous la Troisième République, époque de grande modernisation de Paris qui s'inscrit dans le prolongement du Second Empire, l'administration de la capitale a présenté son activité dans le cadre de diverses Expositions universelles. Elle a ouvert dans leur sillage des musées professionnels ayant des objectifs de formation interne et de promotion de son action, en matière notamment d'hygiène et de santé publique.

3 Après un bref tableau de la situation actuelle connue - dépôts, réserves, certains ensembles bénéficiant peu ou prou du statut de collections patrimoniales aux yeux des entités ou des individualités qui les conservent - il sera procédé à une présentation des premiers musées techniques municipaux. Puis un focus sur la présentation des efforts de la Ville de Paris en matière d'hygiène et d'assainissement permettra d'approfondir la genèse du "Musée municipal de l'Hygiène", lointain ancêtre de l'actuelle "Visite publique des égouts ».

4 Depuis 2015 ont été repérés dans plus de 60 locaux et sites (dépôts, ateliers, centres d'approvisionnement, garages, halls d'entrée, centres d'appel, locaux sociaux, salles de réunion, couloirs, bureaux, sous-sols), dans des directions techniques principalement, de nombreux dispositifs plus ou moins anciens, généralement de petites dimensions, en vitrine ou en présentation directe. Tel est le cas par exemple, du musée du Laboratoire des équipements de la rue, de celui du Service des canaux à Trilbardou (Seine-et-Marne), de la Formation pavage au Centre de maintenance et d'approvisionnement (Direction de la voirie et des déplacements), des bureaux du Service technique des transports automobiles municipaux (Direction de l'immobilier, de la logistique et des transports), de la Maison du jardinage dans le parc de Bercy (Direction des espaces verts et de l'environnement), de l'École de la propreté et de la Visite publique des égouts (Direction de la propreté et de l'eau). D'autres dispositifs ont disparu à la suite de réaménagements ou de transferts, ou sont installés à la faveur d'une nouvelle implantation (Direction de l'urbanisme, Direction des finances et des achats). Certains d'entre eux donnant lieu à des appellations spontanées de "musée ", il s'agit soit d'initiatives personnelles récentes individuelles ou collectives - soit de résidus d'ensembles plus anciens. Dans tous les cas, ce sont bien les traces d'une culture alliant tradition d'innovation et esprit de collection consubstantielles à l'ethos artisanal. Le soubassement historique présenté dans cette étude conforte ces observations empiriques incomplètes; l'intuition que ce sont les vestiges d'un ensemble plus vaste est confirmée par des recherches montrant que l'administration préfectorale a généré une culture d'organisation fondée sur la modernisation de Paris depuis le Second Empire qui a produit de nombreux artefacts ${ }^{2}$. La présentation de son activité dans un but promotionnel et pédagogique s'est faite par le truchement de l'organisation de visites, de sa présence dans diverses Expositions internationales ou universelles ${ }^{3}$, et par l'existence de musées professionnels « industriels » ouverts au public. Nous trouvons les traces de ce type de dispositif dans des textes de nature administrative et dans quelques installations actuelles. 


\section{Brèves histoires de musées}

Capitale d'un pays très centralisé où l'histoire de la ville tend à se confondre avec celle du pays, Paris a tardé à promouvoir son action proprement municipale. Néanmoins, progressivement, un contexte muséal spécifique s'est dessiné à partir des années 1860 . Cela est à mettre en regard de la création concomitante des premiers musées municipaux artistiques, étudiée par Georges Brunel ${ }^{4}$ : «Paris n'a pas eu de musée municipal avant la fin du XIXe siècle. Les musées de l'État [...] suffisaient à la satisfaction des amateurs ». La Ville commandait des œuvres pour la décoration de ses monuments, quelques témoignages laissant penser que la collection des maquettes de ces décors était abritée, depuis la monarchie de Juillet, dans les magasins situés entre le quai Henri-IV et le boulevard Morland ${ }^{5}$. Cette politique municipale fut développée à la faveur d'un remaniement des services en 1867, qui institua la nouvelle Direction des travaux d'architecture, beaux-arts et fêtes, avec à sa tête Victor Baltard (1805-1874), inspecteur supérieur des Beaux-Arts ${ }^{6}$. Paris commença à acheter des peintures et des sculptures lors des Salons annuels puis également, à partir de la fin du siècle, des objets d'art décoratif dans les Salons d'art industriel dans la perspective d'ouvrir des musées municipaux, ainsi que des objets, du mobilier et des œuvres anciennes destinées à présenter le Paris d'antan. Parmi eux, deux établissements, disparus depuis, retiennent notre attention, à la suite de Georges Brunel : le musée des Collections artistiques ou musée d'Auteuil (1887), et celui des Arts appliqués, le premier musée Galliera (1895)

6 Une collection municipale avait commencé à être rassemblée à l'Hôtel de Ville, puis entre le quai Henri-IV et le boulevard Morland (fig. $\left.{ }^{\circ} \mathbf{1}\right)$, tandis qu'une partie était aussi entreposée à partir de 1866, année de son acquisition, dans les combles de l'hôtel Carnavalet, pour ce futur musée destiné à présenter les vestiges du Paris ancien qui disparaissait pour faire place à une ville moderne. «La compensation de ces ravages fut une attention nouvelle portée à l'histoire de la ville et un plan pour sauvegarder des témoignages du passé que l'on était en train d'abolir $»^{8}$. À la suite de longs travaux d'aménagement, le musée-bibliothèque ouvrit ses portes en 1888. En outre, la municipalité décida en 1886 de construire un édifice qui abriterait les richesses artistiques de la Ville et permettrait de les présenter au public : le musée-dépôt d'Auteuil, inauguré en 1887, conçu comme une réserve visitable, devenait ce « résumé du travail de l'administration parisienne au xix siècle ». Il fut fermé au public après la Première Guerre mondiale et ses collections sont rassemblées depuis le milieu des années 1970 au dépôt d'Ivry. Par un concours de circonstances, la Ville disposait en outre, sur la pente de la colline de Chaillot, d'un bâtiment propre à faire un musée 9 . Il fut décidé d'y transporter les collections d'objets d'art de la Ville, ce qui permit l'ouverture du premier musée Galliera en 1895. D'autres musées ouvrirent à la même époque : pour ses collections de Beaux-Arts, le musée du Petit Palais (édifié pour l'Exposition universelle de 1900) en 1902 et, par effet d'entraînement, le musée Cernuschi et la maison de Victor Hugo, respectivement en 1898 et en 1903, à la suite de donations importantes. 


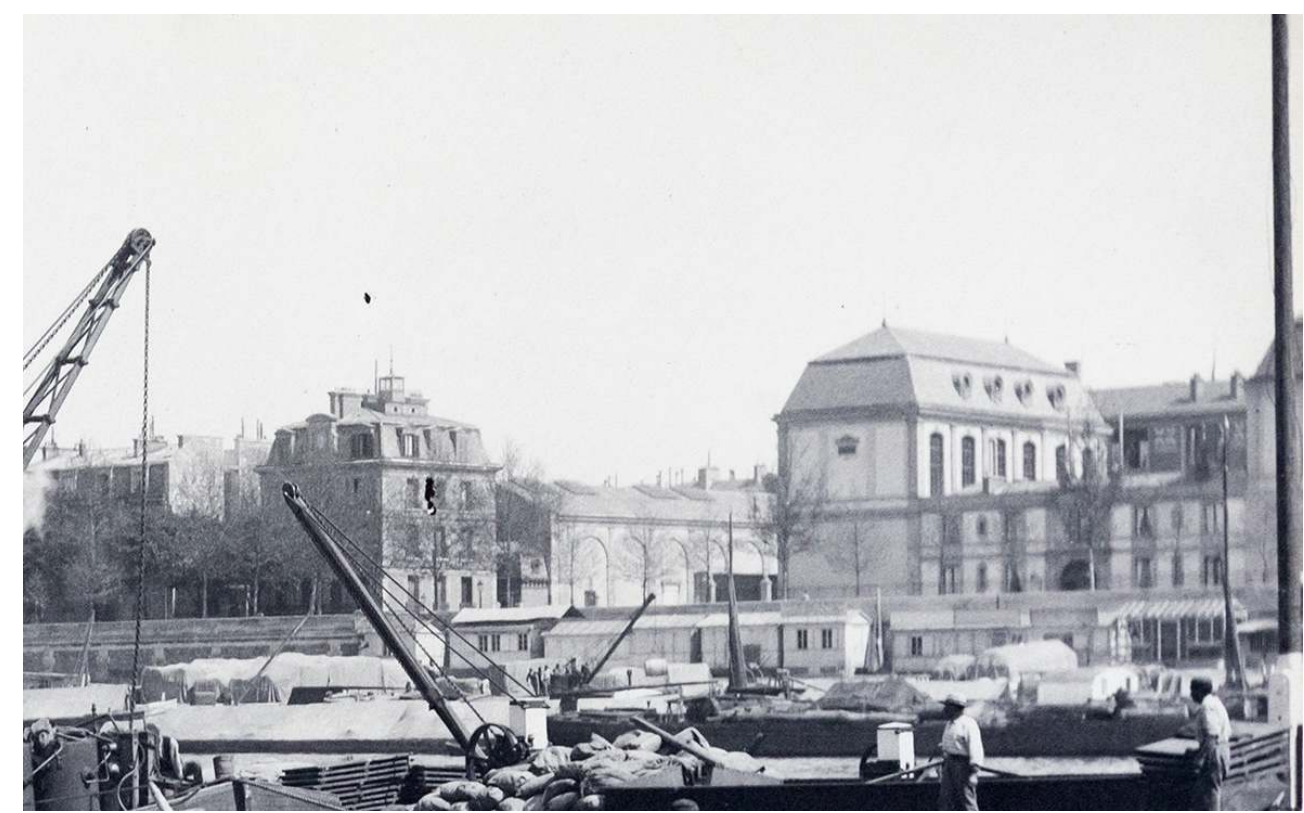

Blanquard, Bateaux près du port aux vins avec le quai Henri-IV dans le fond, photographie (détail), musée Carnavalet, Cabinet des arts graphiques (inv. Ph.73799).

(c) Mairie de Paris/Florence Morisson, MPP, 2018.

Parallèlement, dans un but d'instruction des ouvriers parisiens, ouvrait en 1888 une bibliothèque d'Art industriel dans le faubourg Saint-Antoine grâce à un legs de l'industriel Samuel-Aimé Forney (1819-1879) ${ }^{10}$. De manière concomitante, à une époque où la culture technique et scientifique n'était pas considérée comme inférieure à la culture artistique, d'autres ensembles allaient voir le jour. Grâce à des recherches bibliographiques et diverses informations recueillies sur certains sites, on peut établir une première chronologie de la genèse de projets et du développement des musées professionnels.

\section{Musée pédagogique de la Ville de Paris, 1872}

9 L'Encyclopédie municipale de $1903^{11}$ fait mention d'un Musée pédagogique de la Ville de Paris. Présenté comme une annexe de la Direction de l'enseignement primaire de la Seine, il fut installé à sa création, en 1872, dans le local du magasin scolaire, 36 boulevard HenriIV, puis transféré à l'annexe de l'Hôtel de Ville (rue Lobau) et, en 1894, dans un local situé 47 rue Montmartre. À la fois lieu de conservation et magasin, il se composait d'une bibliothèque, d'un musée proprement dit et d'une «salle des types »:

Le musée comprend des collections très complètes de travaux d'élèves, des écoles maternelles et enfantines, des écoles primaires de garçons et de filles, des cours complémentaires, des écoles primaires supérieures et professionnelles. La salle des types comprend les spécimens de tous les objets en usage dans les écoles de la ville. Tout est catalogué. Le choix étant fait, le magasin scolaire délivre les meubles à l'intéressé.

Dépôt des collections du Service technique de l'assainissement des habitations, après 1889 
11 En 1896, le rapport présenté par Auguste Ranson (1854-1928), président du conseil général de la Seine, au nom de la $6^{\mathrm{e}}$ sous-commission du Budget et du Contrôle (hygiène, eaux, égouts) mentionne ${ }^{12}$ qu'un crédit des travaux à l'entreprise supporte

les frais d'installation, d'agrandissements et de modification du Musée ou si l'on préfère, du Dépôt des Collections du service technique de l'assainissement des habitations. Ce musée, installé dans un ancien hangar dépendant du Dépotoir municipal sis 185 bis, rue d'Allemagne, a été constitué à l'origine par l'ensemble des collections, modèles, dessins et appareils sanitaires qui avaient figuré à l'Exposition universelle de 1889.

\section{Musée de l'Industrie du bois, ou Musée forestier, après 1889}

L'Encyclopédie municipale ${ }^{13}$ mentionne également un musée de l'Industrie du bois à l'école d'arboriculture de Saint-Mandé, école fondée en 1867 par l'ingénieur horticole Alphonse Du Breuil 1811-1890) et, subventionnée par le département, dépendant du Service des promenades. Elle figure en 1901 au budget municipal :

L'école a pour but principal de donner un enseignement pratique et professionnel aux agents et ouvriers municipaux qui se destinent au service des plantations d'alignement; un diplôme de capacité, délivré à la suite d'examens annuels de sortie, atteste la valeur des élèves. Ce diplôme est d'ailleurs exigé pour l'emploi de cantonnier-chef des plantations. L'école est rattachée à la Conservation du bois de Vincennes. Le Musée de l'industrie du bois a été édifié dans l'île de Reuilly avec les matériaux provenant du pavillon des Forêts de l'Exposition de 1889.

Salle d'exposition à l'usine municipale des pavés de bois de Javel, entre 1889 et 1896 ?

Une publication récente consacrée à l'usine municipale des pavés de bois de Javel ${ }^{14}$ fait état d'une salle d'exposition "consacrée à l'étude et aux techniques de fabrication des pavés de bois ». Créée en 1886, l'usine employait alors "cent soixante-dix ouvriers et produisait $20000 \mathrm{~m}^{2}$ de pavés de bois par an, ce qui correspond à $180000 \mathrm{~m}^{2}$ de pavage, de telle sorte que les surfaces couvertes de pavés de bois à Paris dépassaient $2000000 \mathrm{~m}^{2}$ ». Elle connut son apogée vers 1908.

Salle des collections au Laboratoire d'essai des matériaux, avant 1931

Le numéro hors-série de la revue Science et industrie de 1931 reproduit une vue de la salle des collections du laboratoire d'Essai des matériaux, fondé en 1868. Y figurent des échantillons et éprouvettes des matériaux utilisés sur la voirie parisienne dont la solidité et la durabilité sont expérimentées ou contrôlées par le laboratoire.

19 La présentation de la Direction générale des services techniques de Paris dans le supplément de la revue Travaux (édition de 1949), mentionne l'installation et l'ouverture en 1933 d'un petit musée géologique place Denfert-Rochereau, dans le bâtiment de l'Inspection générale des Carrières. Dans ses vitrines sont classés les échantillons des terrains de la région parisienne, des fossiles ainsi qu'une maquette reproduisant les conditions dans lesquelles se présentent les carrières avec leurs dégradations, les consolidations et galeries de surveillance, le bâti et le réseau viaire construits au-dessus. Musée du Laboratoire des équipements de rue (nom pris en 1991)

21 Celui-ci rassemble plusieurs centaines d'objets relatifs à l'éclairage des rues et à la coordination des feux de signalisation, ce qui laisse penser que le rassemblement de ces objets a débuté - après la création dans les années 1860 du Laboratoire de l'inspection du gaz $^{15}$-, avec l'inauguration en 1889 d'une usine électrique municipale dans les sous-sols 
des Halles, usine à laquelle fut associé un laboratoire pour comparer les diverses solutions qui s'offraient alors pour l'éclairage électrique. D'adjonctions en fusions successives (Compagnie parisienne de distribution de l'électricité), depuis les années 1930, ces entités contribuèrent à l'élaboration de la théorie de l'éclairage public, à l'agrément et à l'étalonnage des compteurs électriques, à l'établissement des bases de la régulation de la circulation automobile dans la zone centrale de Paris. Le mobilier en chêne remonte vraisemblablement aux années 1930 (armoires vitrées et grand plateau marqués d'un numéro gravé).

Instruction publique, hygiène, circulation et éclairage, embellissement de Paris sont ainsi mis en valeur par l'administration préfectorale de la Troisième République, par le truchement de ces collections.

Une ambition plus vaste avait été formulée par Eugène Viollet-le-Duc (1814-1879) en 1877 devant le conseil municipal, au nom de la $5^{\mathrm{e}}$ commission (Architecture et Beaux-Arts) qu'il présidait, dans un rapport ${ }^{16}$ concernant la participation des services municipaux à l'Exposition universelle de 1878. Déplorant que l'administration parisienne ne se soit pas présentée en tant que telle à l'Exposition de 1867, l'architecte préconisait la création d'un «Musée municipal industriel » et demandait l'attribution de $3430 \mathrm{~m}^{2}$ dans l'un des angles de la partie du Palais voisine de l'École militaire. Après la clôture de l'Exposition,

cette somme ne serait pas improductive pour la ville de Paris [...]. Une grande partie des objets auxquels elle s'applique présente un caractère d'utilité permanente ; sans compter les engins exposés qui prendront leur place dans les services auxquels ils sont affectés, les objets d'art, les modèles, les tracés graphiques prendront place dans les collections de la Ville, qui, jusqu'à présent, ne possède d'autre Musée que celui de l'hôtel Carnavalet, dont la destination est toute spéciale, mais cette lacune fâcheuse doit être comblée. Nous avons entendu dire, notamment au temps de l'Empire, sous le régime duquel on avait demandé la formation d'un Musée municipal à plusieurs reprises, que les Musées de l'État étant installés à Paris, il n'y avait pas lieu de faire un double emploi en ouvrant un Musée de la Ville de Paris. Mais alors on ne comprenait pas ou on ne voulait pas comprendre l'esprit de ces demandes. Il ne s'agissait pas d'ouvrir un Musée qui fût une doublure, à coup sûr très réduite, du Musée du Louvre. Il s'agissait de créer un Musée qui fût spécialement destiné à fournir aux diverses industries de Paris, des éléments précieux de travail. Le Louvre rejette [...] tout ce qui présente un caractère industriel. [...] La création d'un Musée municipal industriel est donc une œuvre utile, et il n'est pas douteux que si un noyau était formé, les dons affluassent. Nous connaissons des amateurs, parisiens de fait ou parisiens de cœur, qui seraient fiers de laisser leurs collections à la Ville de Paris ; mais il faut un local, un local spécial, propre au rangement de ces objets, propre à l'étude. L'exposition de la Ville de Paris peut composer ce noyau.

\section{Montrer l'organisme de la ville, le rôle dévolu aux pavillons de la Ville de Paris lors des Expositions universelles}

24 L'action municipale en la matière fut plus modeste, puisqu'elle concentra ses efforts sur les activités proprement municipales, en particulier sur l'hygiène et l'assainissement. Après quelques tentatives, le Paris du Second Empire et de la Troisième République connut une modernisation d'une ampleur jusqu'alors inégalée, portée par le corps des ingénieurs des Ponts et Chaussées, sur les conditions de laquelle nous ne nous 
appesantirons pas. Le projet d'en faire la plus belle ville du monde, capitale de l'Empire, cité de tous les Français selon la terminologie haussmannienne, s'est notamment articulé avec des considérations techniques relatives à l'hygiène et à la santé. La mortalité due à de terribles épidémies de choléra et de typhoïde, à la tuberculose, est au centre des préoccupations des élites techniques et des responsables de l'administration.

Les questions de la distribution de l'eau et de l'assainissement de la cité sont parmi les plus urgentes à régler. L'aération, la ventilation, la lutte contre la poussière mobilisent la recherche et les capacités d'innovation des ingénieurs et des entreprises. L'aspiration au confort et au bien-être, portée par les nouvelles couches sociales urbaines, pose les problèmes de distribution des énergies aux particuliers : chauffage, électricité, téléphone. La question des déplacements, de la circulation des piétons, voitures particulières et transports en commun, reste un problème obsédant pour la vie quotidienne et l'essor de l'économie ${ }^{17}$.

À l'heure de la multiplication des contacts physiques dans une ville de plus en plus peuplée, la collaboration sanitaire entre des instances d'étude ou de régulation de plus en plus nombreuses à Paris comme en Europe est perçue comme un impératif. En 1851, réunissant douze pays d'Europe, se tient à Paris la première conférence sanitaire internationale ${ }^{18}$. Le congrès international d'Hygiène, organisé à Paris en 1878 pendant l'Exposition universelle, recommande l'évacuation totale des matières à l'égout et leur épandage ${ }^{19}$. Sont notamment proposées aux congressistes une visite de l'usine élévatoire de Clichy et de la presqu'île de Gennevilliers ainsi que celle du grand collecteur et de l'égout qui s'étend sous la rue de Rivoli. Après une période au cours de laquelle prévaut le projet haussmannien d'un égout propre - ne recevant que les eaux issues des rues, à l'exclusion des effluents évacués des fosses par des vidangeurs - puis une phase facultative du tout-à-l'égout de 1885 à 1894 (loi du 10 juillet 1894), ce n'est guère qu'à partir de 1905 que la bataille contre le " péril fécal » paraît gagnée ${ }^{20}$.

Cette vision métaphorique de la grande ville assimilée à un organisme vivant, conception qui trouve vraisemblablement son origine au $\mathrm{xvIII}^{\mathrm{e}}$ siècle, connait un développement important dans la seconde moitié du XIX ${ }^{\mathrm{e}}$ siècle, avec notamment l'ouvrage de Maxime Du Camp (1822-1894), Paris, ses organes, ses fonctions, sa vie $e^{21}$. Viollet-le-Duc la reprend à son compte dans son rapport pour l'Exposition universelle de 1878 (voir supra). Quelques décennies plus tard, l'Encyclopédie municipale la développe avec lyrisme, insistant sur le contraste entre le visible et l'invisible, le dessus et le dessous ${ }^{22}$ (fig. ${ }^{\circ}$ ). 
Figure 2

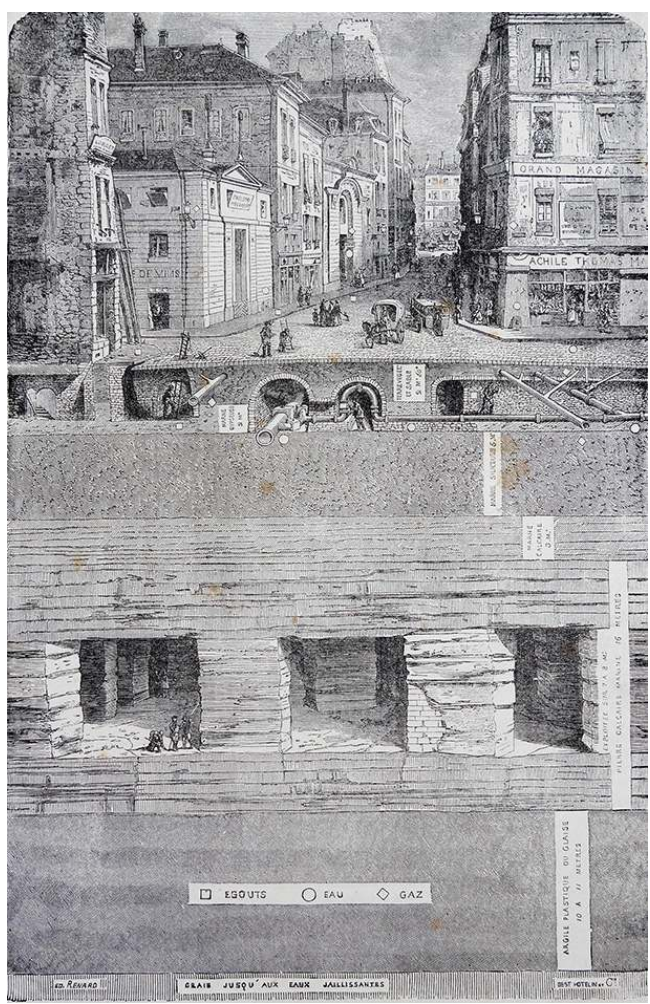

Ed. Renard, Le dessus et le dessous de Paris, bois gravé, F. Roy éditeur, musée Carnavalet, Cabinet des arts graphiques (inv. G.36131).

(c)Mairie de Paris/ Florence Morisson, MPP, 2018.

La construction des égouts fera l'objet de descriptions émerveillées, mises en valeur par la parution dans la presse à grand tirage, à partir de 1858, d'articles illustrés de grandes planches de bois gravés, puis en 1865, par un reportage photographique effectué par Nadar (1820-1910) ${ }^{23}$ (fig. $\left.{ }^{\circ 3}\right)$. « Tous les journaux ont parlé de l'entreprise de Nadar, qui photographie les égouts de Paris, comme il a déjà photographié les catacombes à l'aide de la lumière électrique ». Dès l'Exposition universelle de 1867, la Ville de Paris ouvre les égouts à la curiosité du public en organisant des visites destinées à promouvoir cet équipement. Elle renouvelle l'expérience en 1878 (voir supra). Ces « excursions de plaisir » souterraines entièrement balisées se poursuivent sur un itinéraire éclairé à l'électricité et sous la conduite d'égoutiers. Recommandée par les guides touristiques qui vantent sa valeur "instructive et intéressante», la promenade souterraine ménage quelques émotions fortes ${ }^{24}$. 


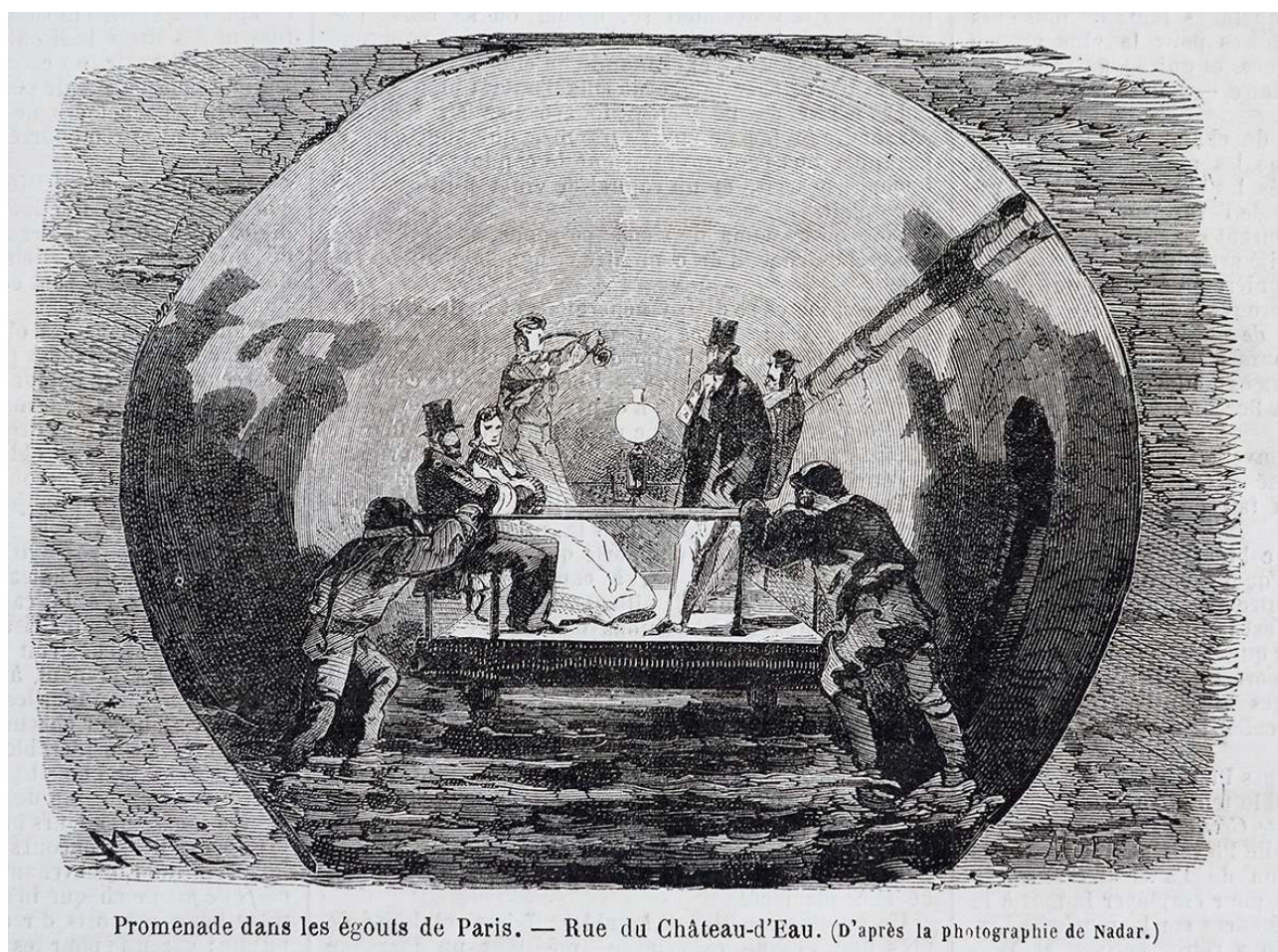

Morin, Promenade dans les égouts de Paris. Rue du Château-d'Eau (d'après la photographie de Nadar), bois gravé, musée Carnavalet, Cabinet des arts graphiques (inv. G.36113).

(c) Mairie de Paris/ Florence Morisson, MPP, 2018.

\section{L'Encyclopédie municipale précise, en $1903^{25}$ :}

Depuis l'exposition de 1867, des visites publiques des égouts ont lieu, deux fois par mois, pendant la saison d'été. Elles se faisaient, autrefois, entre les places du Châtelet (fig. $\left.{ }^{\circ} \mathbf{4}\right)$ et de la Madeleine. Deux groupes d'une centaine de visiteurs partaient des points extrêmes et se rencontraient sous la place de la Concorde. Le trajet s'effectuait par wagons sur rails dans les collecteurs Sébastopol et Rivoli, et par bateaux-vannes spécialement aménagés à cet effet, dans le collecteur d'Asnières sous la rue Royale. En raison de la construction de la ligne métropolitaine qui emprunte la rue de Rivoli, l'égout-salon de cette rue a été démoli, et l'itinéraire des visites d'égouts a dû être modifié ; il comprend actuellement le parcours entre les Arts et Métiers et le quai du Louvre, par les collecteurs du Centre ou des PetitsChamps et Sébastopol; les wagons circulent entre la rue Saint-Martin et le Châtelet; les bateaux vont du Châtelet au Louvre et vice-versa. Les gares extrêmes sont éclairées à la lumière électrique, des plaques indicatives renseignent à chaque instant les visiteurs sur le parcours effectué ; enfin, l'ancienne traction à bras des wagonnets et des bateaux a été remplacée par la traction électrique. Les visites ont lieu les $2^{\mathrm{e}}$ et $3^{\mathrm{e}}$ mercredis de chaque mois. Pour obtenir des cartes d'autorisation, s'adresser à la Direction administrative de la voie publique, à l'Hôtel de Ville, ou au Service technique des égouts et de l'assainissement, 9, place de l'Hôtel-de-Ville. 


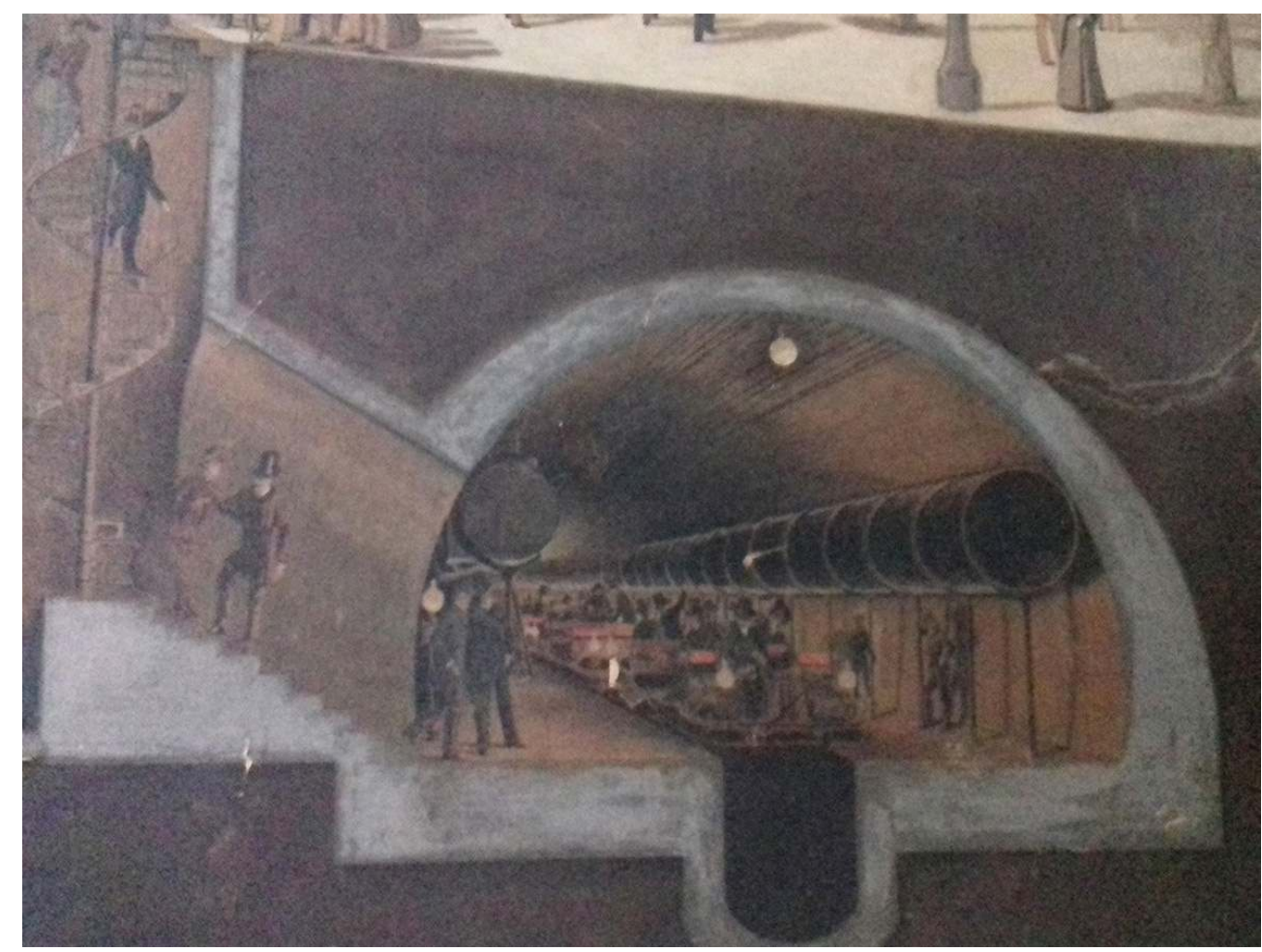

Anonyme, Entrée des collecteurs, place du Châtelet, aquarelle (détail), Exposition spéciale de la Ville de Paris, Exposition universelle de 1889, cat.75, Archives de Paris, VO3 1219.

(c) Mairie de Paris/ Miriam Simon, MPP, 2018.

L'Annuaire municipal de 1925 décrit le même parcours ${ }^{26}$. Une affiche de 1968 propose la visite depuis la place de la Concorde (fig. ${ }^{\circ}$ ). En 1975, ce parcours sera remplacé par un "musée » installé dans l'usine Alma, au niveau du départ du siphon, sur un site en exploitation visitable à pied dans lequel des vitrines présentent une histoire des égouts au moyen d'objets trouvés dans les égouts, de modèles réduits, d'outils et de matériel de curage. En 1989, la Visite publique des égouts est réaménagée, et enrichie depuis de panneaux pédagogiques au gré de l'apport d'expositions diverses. Actuellement, la visite se fait toujours sous la conduite d'égoutiers ${ }^{27}$. 


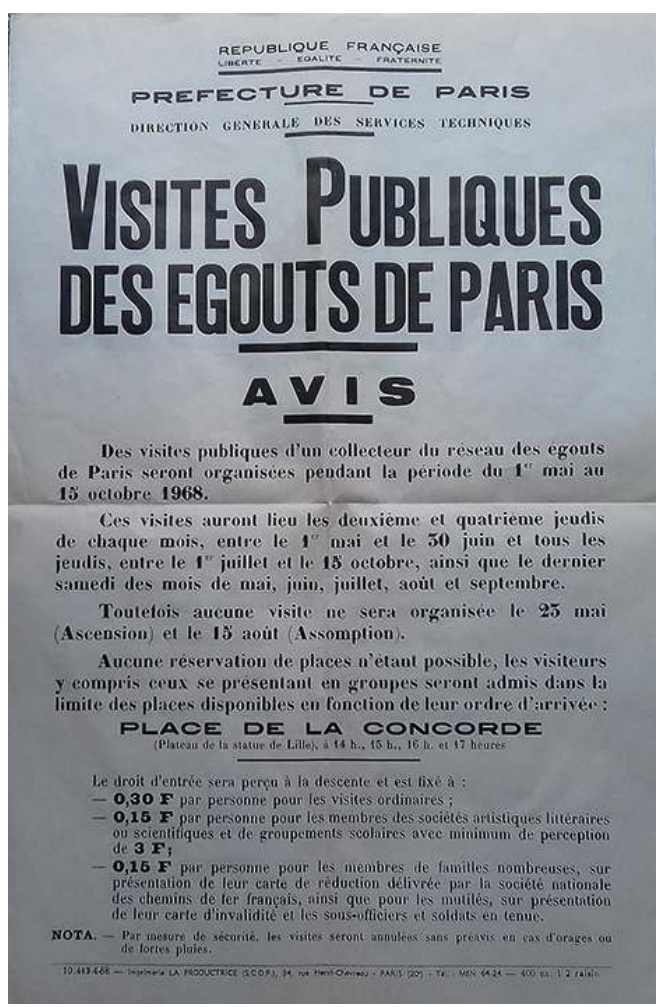

Avis de visite des égouts, imprimé, 1968, Direction de la propreté et de l'eau.

(c) Mairie de Paris/ Miriam Simon, MPP, 2018.

Parallèlement, Paris fut le siège de plusieurs Expositions universelles, vitrines de modernité, grands moments de compétition entre nations industrialisées. Après celle de 1867, dont le programme centré sur le travail avait été confié à l'ingénieur Frédéric Le Play (1806-1882), la Ville de Paris eut à cœur de présenter sa propre modernisation avec un souci pédagogique évident, au moyen de maquettes, modèles réduits, dessins, aquarelles, photographies, graphiques, panoplies d'outils, mannequins en tenue, équipements en taille réelle.

31 Dans son rapport extraordinairement intéressant pour l'Exposition universelle de 1878, qui mérite d'être amplement retranscrit, Eugène Viollet-le-Duc (voir supra) s'interroge : la ville de Paris doit-elle

se borner à laisser voir aux étrangers ses monuments, ses parcs, ses voies si habilement percées, ses quais, ses ponts ? N'a-t-elle pas à montrer l'organisme [...] de la grande ville, comment procède son édilité, par quelle suite d'efforts et de travaux accumulés sont obtenus ces résultats, conséquences de labeurs persistants, suivis avec un esprit de méthode ? [...] Mais aussi, l'étranger est assez disposé à croire que ces biens sont une faveur toute spéciale, due au climat, à la situation géographique, à des causes inappréciables, à un hasard heureux, si l'on veut. Il ne peut savoir, par quelle suite de longs efforts, cette ville est arrivée à être à la fois, et la capitale du travail et la capitale des plaisirs, des distractions intellectuelles et des satisfactions matérielles. [...] En 1867, l'Administration de la ville de Paris ne participa pas à l'Exposition et se contenta d'éblouir les étrangers par les résultats acquis, sans les inviter à se rendre compte des moyens à l'aide desquels ces résultats étaient obtenus. Elle eut tort, certainement. [...] M. le Préfet de la Seine a [...] composé une Commission chargée d'étudier les éléments propres à fournir à 
l'Administration municipale et départementale l'occasion de faire une Exposition digne de la ville de Paris, et dans laquelle figureraient les progrès réalisés chaque jour dans les diverses branches de cette Administration, les nombreux encouragements donnés à l'art et au développement de l'instruction publique, les modèles de l'organisme caché de la grande ville, tout ce qui concerne la salubrité et l'assistance publique, etc. [...] La Commission administrative a pensé, tout d'abord, qu'il fallait ne pas scinder cette exposition, mais, au contraire, l'organiser en un corps spécial et distinct, qui permît de présenter dans leur ensemble les résultats obtenus depuis 1867.

Une importante section fut installée au cœur de la grande galerie, au Champ de Mars, complétée par une annexe des machines installée près du pont de l'Alma (fig. $\left.{ }^{\circ} \mathbf{6}\right)$. La surface, de plus de $3500 \mathrm{~m}^{2}$, était répartie entre les services participants : Administration générale, Enseignement, Beaux-Arts, Travaux historiques, Promenades et Plantations, Plan de Paris, Voie publique, Architecture, Assistance publique, Eaux et Égouts, Préfecture de Police, la Direction de l'enseignement et la Direction des eaux et égouts se taillant la part du lion parmi les services techniques, avec plus de $600 \mathrm{~m}^{2}$ chacune.

Figure 6

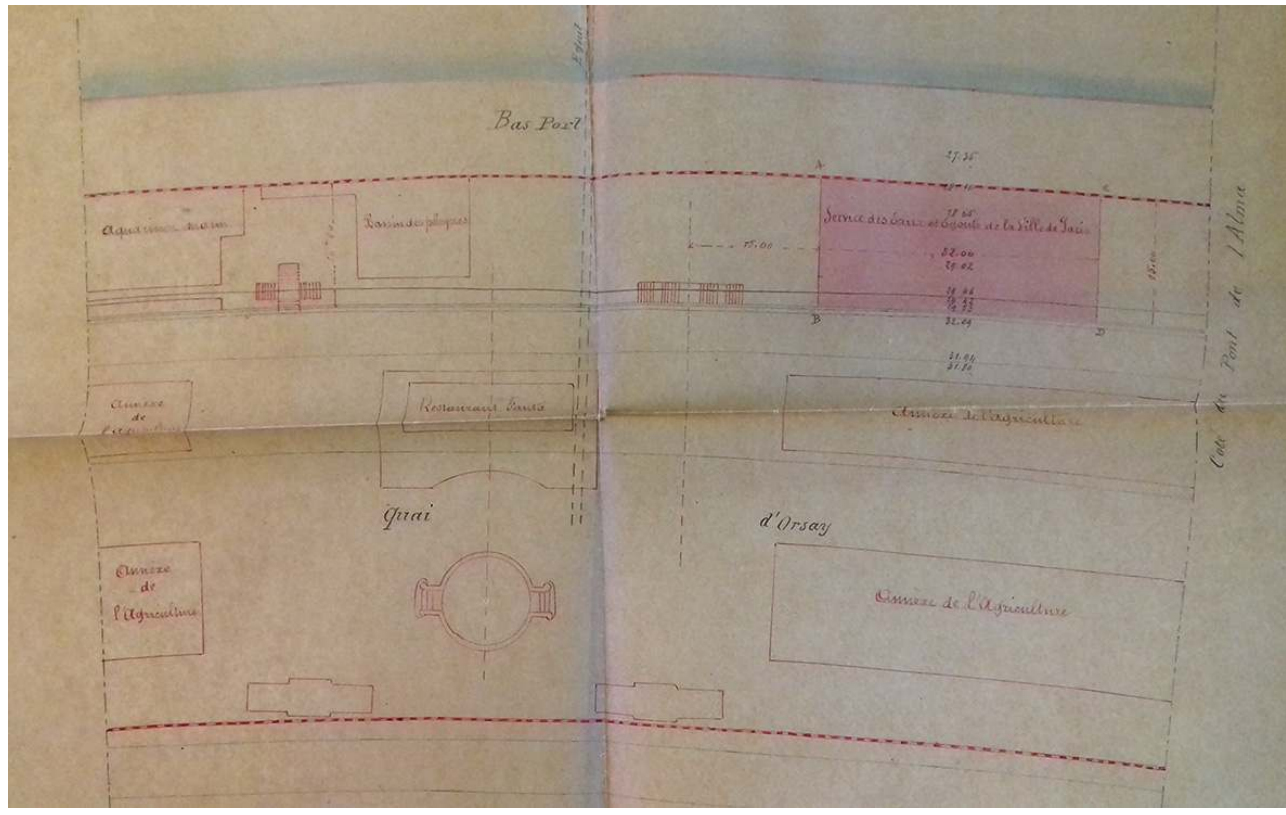

Duval, Plan des installations de l'Exposition de 1878 près du pont de l'Alma, encre de Chine et aquarelle (détail), Archives de Paris, VO3 1219

(c) Mairie de Paris/ Miriam Simon, MPP, 2018.

La dépense (700 000 francs) représentait le prix des travaux nécessaires à la réunion, et surtout à la confection des objets. Une partie fut imputée directement sur les crédits dont disposaient plusieurs des services participants, comme par exemple

le modèle de l'Hôtel de Ville, partie des plans, cartes et albums qui seront exposés par la Direction des eaux et égouts, la restauration et l'encadrement d'un certain nombre d'anciens tableaux et d'anciennes tapisseries, l'exécution des photographies de l'ancien et du nouveau Paris, la confection de types et de modèles et du matériel des établissements scolaires ${ }^{28}$.

La Revue scientifique de la France et de l'étranger présentait l'exposition de la Direction des eaux et égouts en rendant un hommage appuyé à l'ingénieur Eugène Belgrand (1810-1878), décédé cette même année, dont elle fait le héros de l'égout moderne ${ }^{29}$, lui 
qui, « en 1854, traçait de main de maître le programme de l'alimentation d'eau et de l'assainissement de Paris ». L'article détaille un programme très pédagogique :

Cette œuvre se divise en quatre parties, car le fonctionnement du Service des eaux et égouts comprend quatre opérations successives et distinctes. Elles consistent : 1 . À assurer les ressources nécessaires à la distribution c'est-à-dire à amener chaque jour, et emmagasiner à niveau convenable, une quantité d'eau suffisante et de bonne qualité. 2. À distribuer cette eau dans chaque rue pour le service public: dans chaque maison et à chaque étage pour le service privé. 3. À l'évacuer ensuite, avec les eaux pluviales, à mesure que la consommation la restitue, souillée par les usages domestiques et industriels. 4. À épurer cette eau à la sortie des égouts, à la régénérer en quelque sorte avant de la laisser faire retour au fleuve. Ces quatre opérations correspondent dans l'exposition de la ville, aux quatre sections suivantes: 1. Machines, dérivateurs et réservoirs 2. Réseau des conduites de distribution 3. Réseau des égouts et moyens de curage 4. Utilisation agricole des eaux d'égout.

Dessins, photographies, modèles réduits furent réalisés à cette occasion, tandis que l'annexe présentait le gros outillage et divers spécimens d'ouvrages en vraie grandeur. Une attention particulière est portée à la présentation de l'utilisation agricole des eaux d'égouts : tableaux graphiques émanant du Service d'utilisation agricole des eaux d'égout - organisé en 1867 et confié au polytechnicien Adolphe Mille (1812-1894), l'un des ardents promoteurs du tout-à-l'égout et de l'épandage agricole - aquarelles, modèles, planches et documents statistiques, échantillons d'eau d'égout naturelle et d'eau de la nappe et de produits agricoles de la plaine de Gennevilliers (fig. ${ }^{\circ}$ ).

\section{Figure 7}

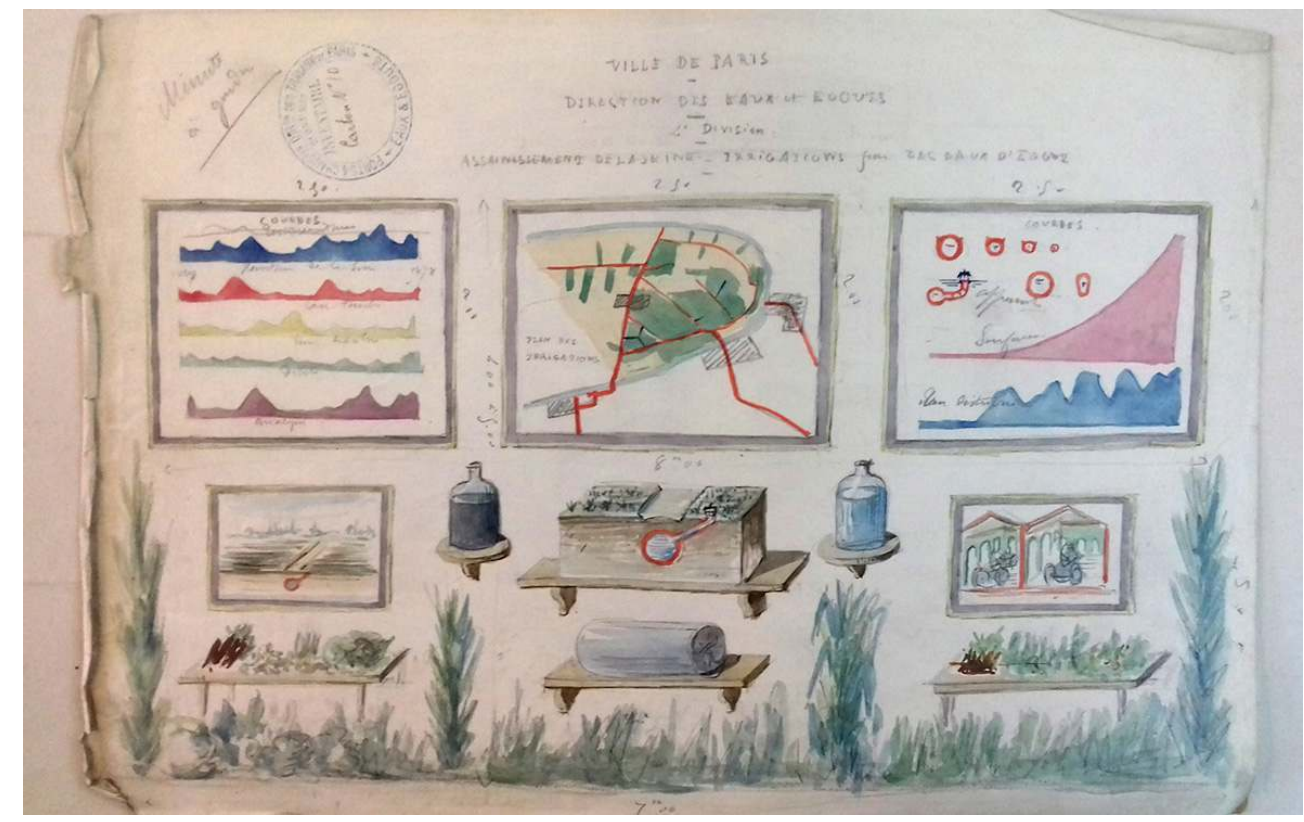

Anonyme, Projet de disposition de l'exposition du Service d'utilisation agricole des eaux d'égout, aquarelle, Archives de Paris, VO3 1219.

(c) Mairie de Paris/ Miriam Simon, MPP, 2018.

Sont également présentés les canaux et la voirie (effluents issus des fosses) : plan général avec courbe de niveau et profil de long, aquarelles, modèles des portes d'écluses, albums statistiques et courbes représentatives du tonnage et des recettes du dépotoir municipal de La Villette («Seule de toutes les capitales de l'Europe, Paris a conservé le système des 
fosses fixes pour la plus grande partie de ses maisons »), voirie de Bondy, ventilation, gaz brûlés dans des chaudières, jardins soignés autour de l'établissement (fig. ${ }^{\circ 8}$ ).

Figure 8

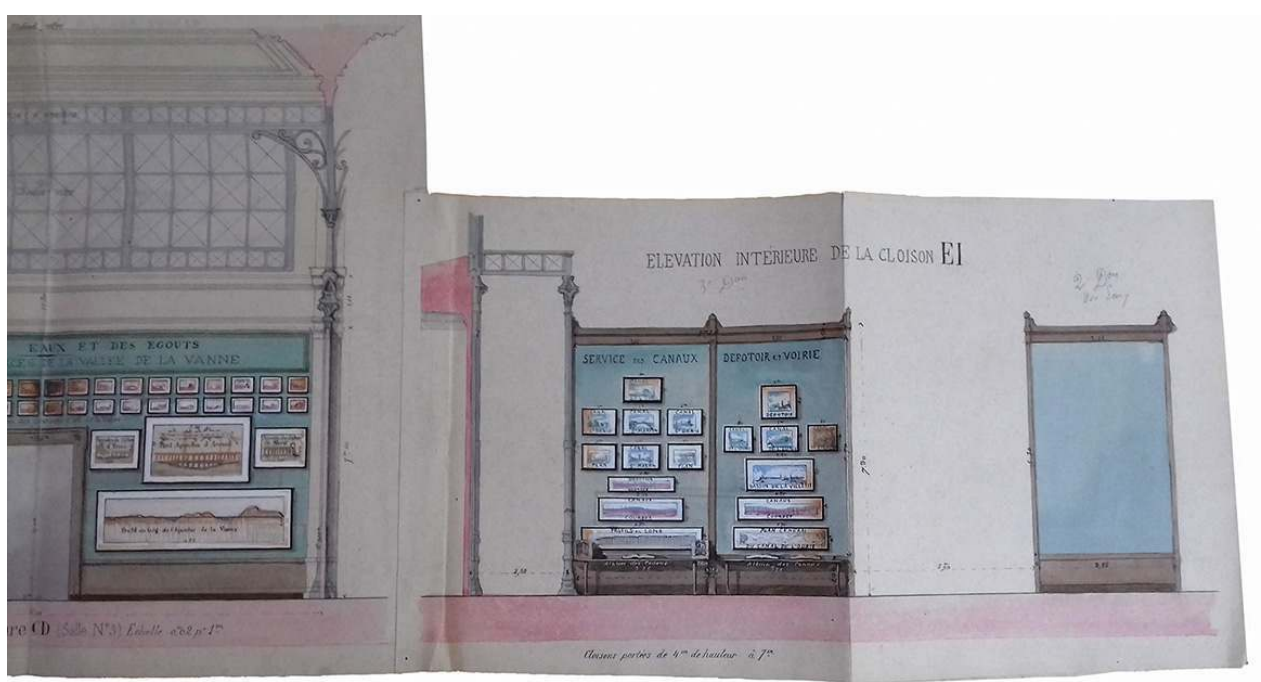

Bouvard, Projet de disposition de l'exposition du Service des canaux et de la voirie, aquarelle (détail), Archives de Paris, V03 1219.

(c) Mairie de Paris/ Miriam Simon, MPP, 2018.

Il en alla de même en 1889, lors de la célébration du centenaire de la Révolution française et de la République au Champ-de-Mars, sur l'esplanade des Invalides, la colline de Chaillot et les rives de la Seine, manifestation qui connut un immense succès avec plus de 28 millions de visiteurs. La Ville de Paris présenta alors l'exposition de tous ses services dans deux pavillons dessinés par Joseph Bouvard (1840-1920), architecte de la Ville de Paris, construits dans le jardin central du palais des Expositions diverses et se faisant face de chaque côté de la grande allée. L'un des deux présentait les services relevant de la Direction des travaux de Paris $^{30}$ (fig. ${ }^{\circ}$ 9). De nouveau, sur une surface totale de près de $1850 \mathrm{~m}^{2}$, l'assainissement était mis à l'honneur, avec l'enseignement primaire. Le clou en était la pédagogie de l'assainissement de l'habitation, par la présentation de deux maisons-types, la maison salubre et la maison insalubre, avec adjonction d'un branchement particulier de l'égout et d'un réservoir de chasse sur l'égout, enrichies de modèles et de dessins, le tout conçu par l'ingénieur Alfred Durand-Claye (1841-1888) ${ }^{31}$. 


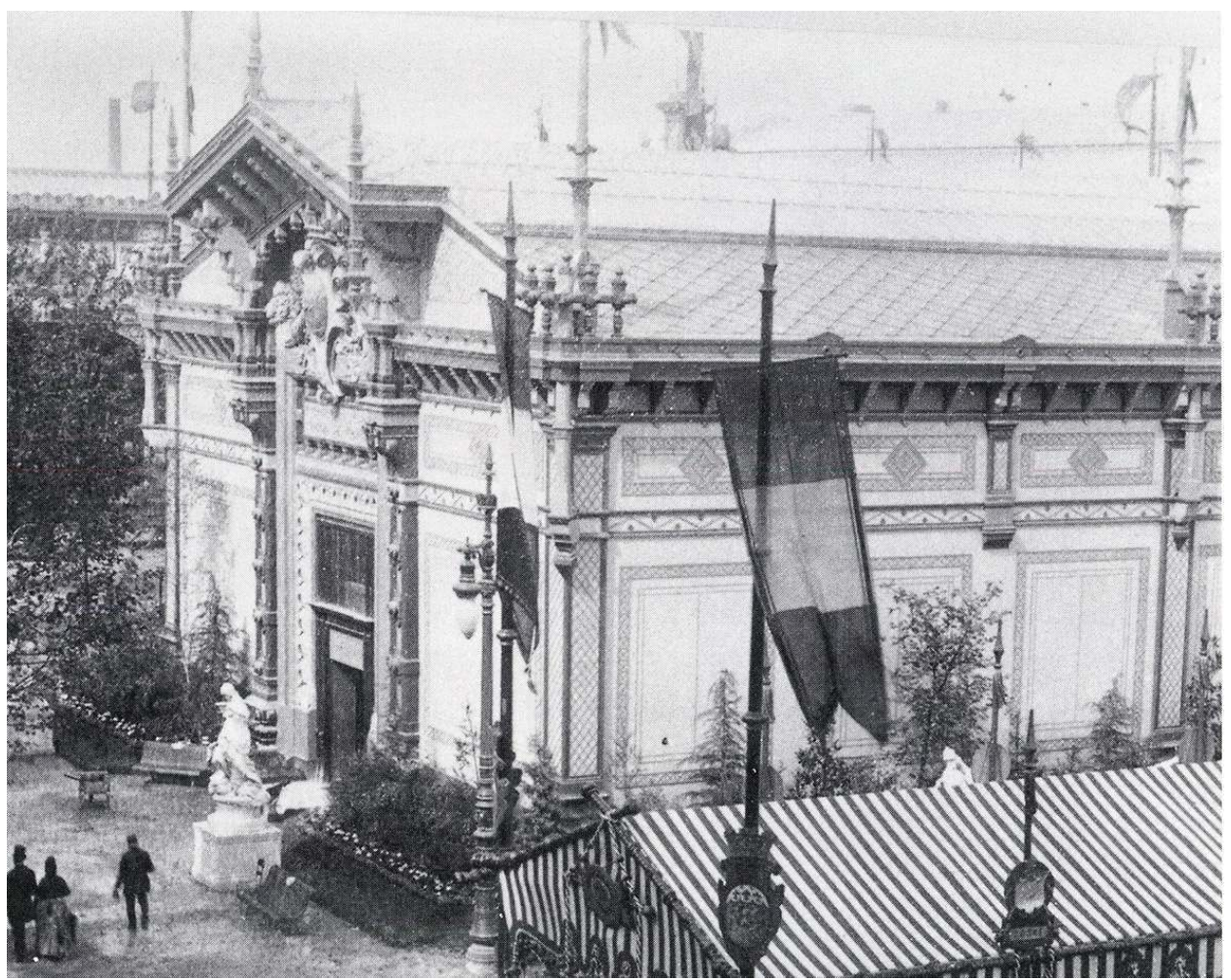

Hippolyte Blanquard, Façade principale des pavillons de l'Exposition spéciale de la Ville de Paris par l'architecte Bouvard, photographie, collection particulière (reproduit avec l'autorisation des commissaires dans le catalogue Quand Paris dansait avec Marianne, Paris, 1989, cat.165, repr. p.238

(c) Mairie de Paris/ Florence Morisson, MPP, 2018.

L'administration parisienne réédita l'opération en 1900 en édifiant un pavillon qui reproduisait à l'identique l'ancien Hôtel de Ville disparu dans les flammes en 1871 (fig.` 10), et était complété par la présentation d'équipements grandeur nature dans une annexe en bord de Seine, cours la Reine ${ }^{32}$. Dans le sous-sol étaient données des conférences et projections lumineuses ayant pour objet les services d'hygiène, d'assainissement et d'alimentation. Tous les travaux d'ameublement et d'installation (vitrines, etc.) furent exécutés par les ouvriers du refuge Nicolas-Flamel (Assistance par le Travail), et les travaux photographiques et stéréoscopiques par les services de la Direction des affaires municipales. 


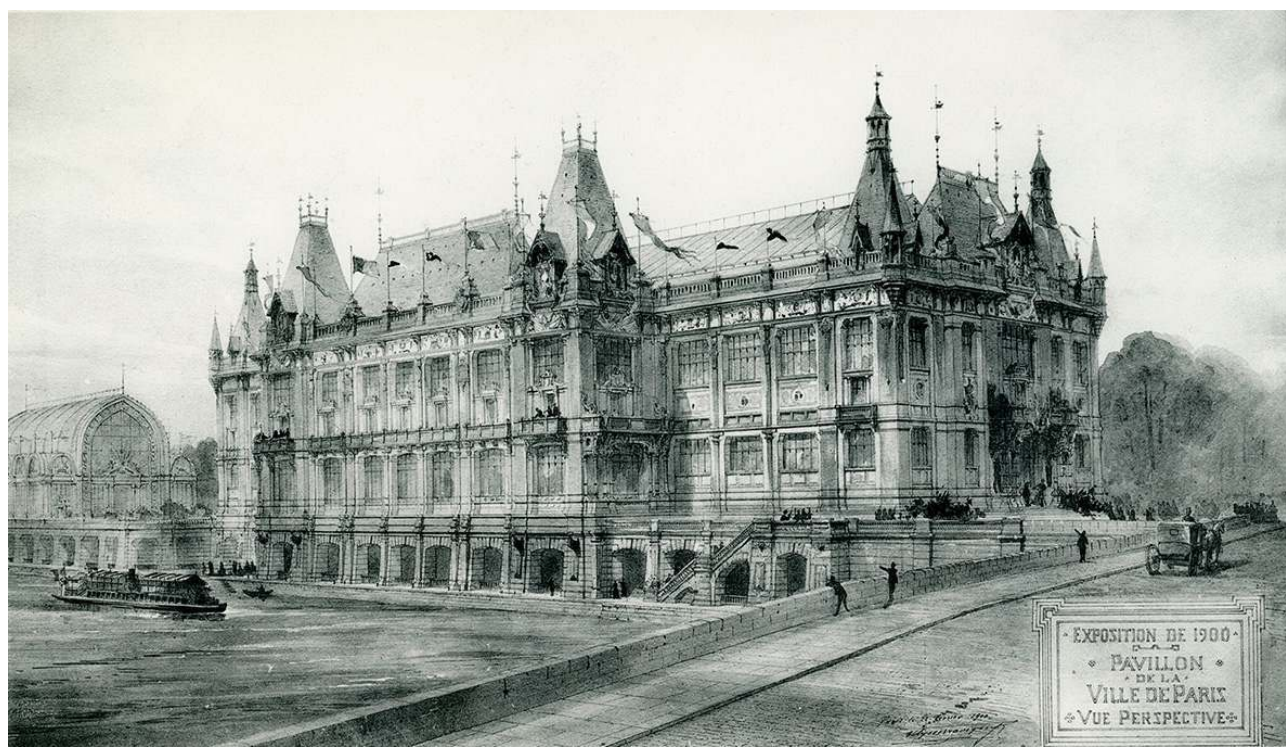

Ulysse Gravigny, del., Berthaud Frères, phot., Exposition de 1900. Pavillon de la Ville de Paris, vue perspective, 1900, Frontispice du catalogue de l'Exposition spéciale de la Ville de Paris et du Département de la Seine.

(c) Mairie de Paris/ Florence Morisson, MPP, 2018.

Concernant les égouts et l'assainissement, la Direction des affaires départementales présentait, au sein du Service technique des eaux et de l'assainissement ${ }^{33}$, le Service des égouts (construction, curage et entretien) et le Service de l'assainissement (assainissement de la Seine, épuration et utilisation agricole des eaux d'égout) par des aquarelles, ouvrages, statistiques, panoplies d'outils, ainsi que de nombreux modèles réduits, retraçant ainsi une histoire de la construction des égouts dans Paris depuis 1801, leur présentation en 1900, exposant les siphons, les usines de relèvement des eaux d'égouts nécessaires à l'irrigation agricole, le matériel de curage et d'entretien. Plusieurs de ces objets appartiennent vraisemblablement à la collection de modèles réduits de l'actuel Service technique de l'eau et de l'assainissement ${ }^{34}$ (fig. $\left.{ }^{\circ} 11\right)$. De grandes aquarelles présentant les différents types d'égouts, retrouvées récemment, ont été transférées au Service des archives. 


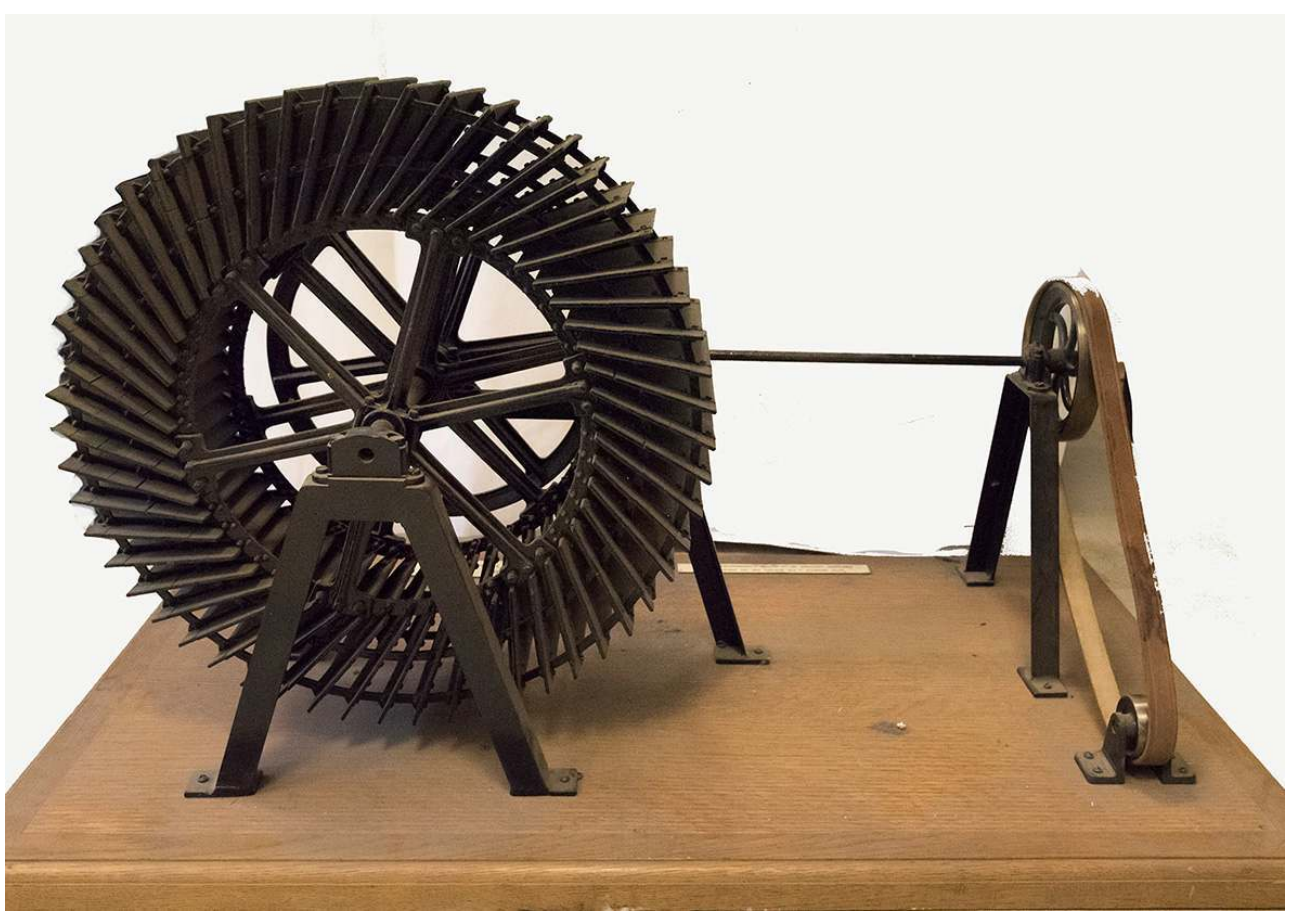

Anonyme, Modèle réduit de la roue de l'usine de Mazas, métal et bois, DPE.

(c) Mairie de Paris/ Florence Morisson, MPP, 2018.

Enfin, la thématique de la salubrité fit également l'objet d'une présentation lors de l'Exposition internationale de 1937, dans le pavillon de la Ville de Paris ${ }^{35}$ et celui de l'Hygiène qui mettaient notamment en valeur la lutte contre les taudis de la capitale avec des maquettes d'îlots insalubres et d'îlots de la Zone ${ }^{36}$ (fig. $\left.{ }^{\circ} 12\right)$. En outre, Paris ne dédaigna pas de valoriser sa modernisation en participant à d'autres Expositions internationales (notamment Vienne en 1873, Londres en 1874, Amsterdam en 1886, avec catalogues, et New York en 1939), ou en présentant à la caserne Lobau, en 1886, une exposition d'hygiène entièrement consacrée à l'assainissement de la maison. 
Figure 12

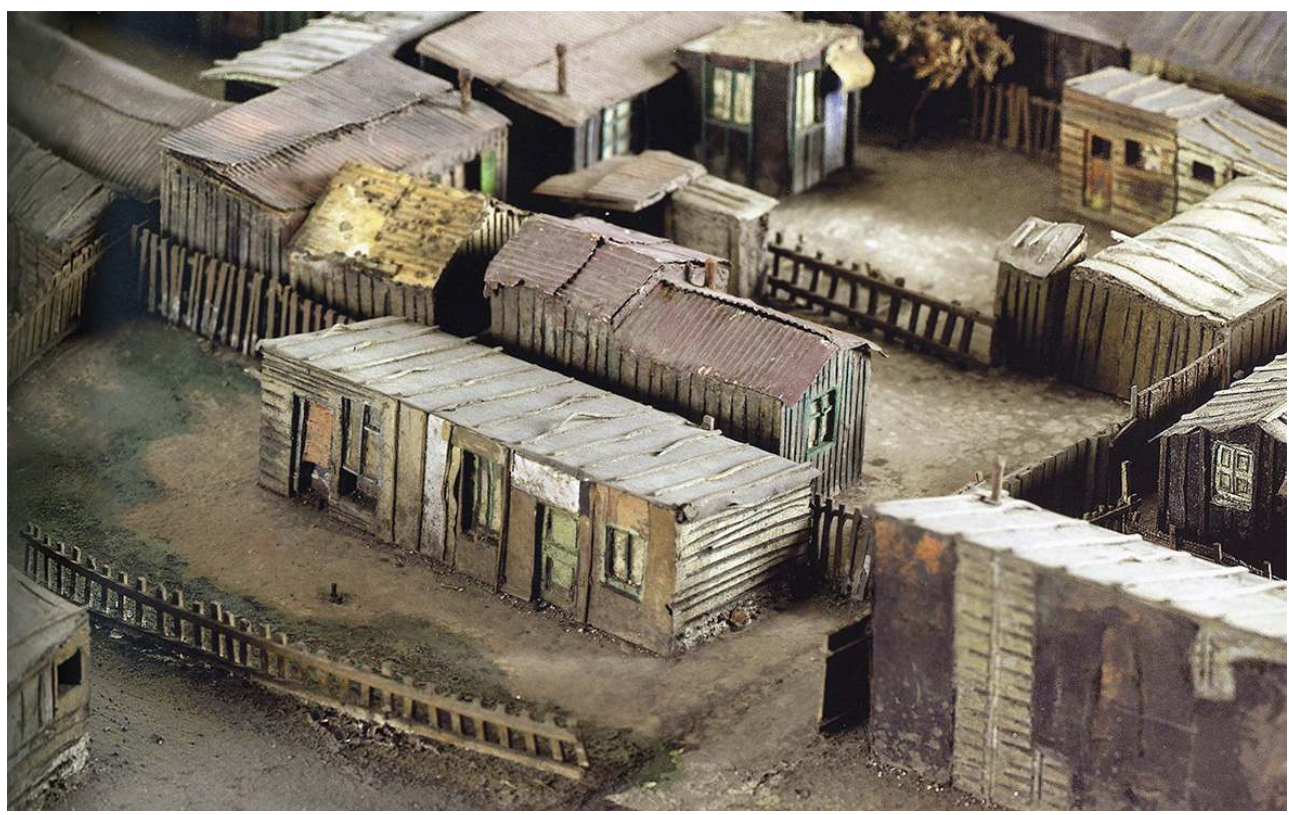

Maquette au 1/100e (détail), Studio E.P.O.C., La Zone près de la porte de Clignancourt, 1937, musée Carnavalet (PM.83), reproduite avec l'autorisation de la commissaire dans le catalogue Le peuple de Paris au XIXe siècle, cat. 23, repr. p. 55, Paris, 2011.

(c) Mairie de Paris/ Florence Morisson, MPP, 2018.

\section{Le musée de l'Hygiène : de la propagande à l'éducation}

41 À travers ces expositions spéciales, nous percevons une conception totalisante de l'hygiène, que l'on retrouve dans le musée municipal d'Hygiène tel qu'il se développe en deux temps correspondant à deux lieux. Les sources dont nous disposons pour procéder à sa reconstitution sont les nombreux rapports présentés devant le conseil municipal entre 1896 et $1912^{37}$, étayés par l'Encyclopédie municipale et des annuaires municipaux.

\section{Au dépotoir de La Villette, prémices d'un Musée sanitaire}

$42 \quad$ (fig. $\left.{ }^{\circ} 13\right)$ 


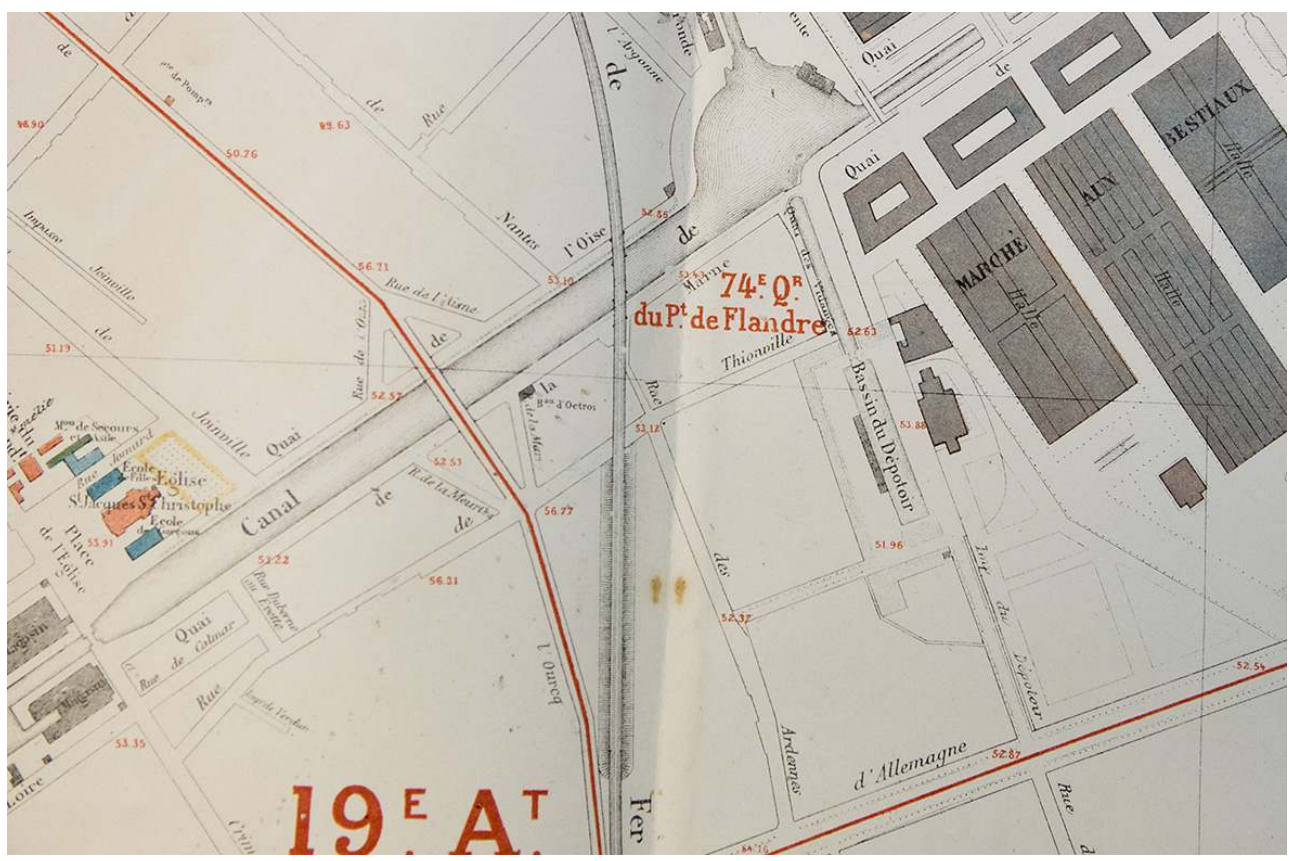

Détail de l'Atlas municipal des 20 arrondissements de Paris, 19e arrondissement, imprimé, 1871.

(C) Mairie de Paris/ Florence Morisson, MPP, 2018.

Un premier rapport, présenté en 1896 par Auguste Ranson, décrit un « Musée ou si l'on préfère [un] Dépôt des Collections du Service technique de l'assainissement des habitations ", installé dans un ancien hangar dépendant du dépotoir municipal, 185 bis rue d'Allemagne ${ }^{38}$, dans une zone industrialisée en raison de sa proximité immédiate avec les canaux de l'Ourcq et de Saint-Denis reliés au bassin de la Villette par la gare circulaire située au carrefour des Trois Canaux. Destiné à remplacer définitivement la voirie de Montfaucon $^{39}$ (fig. ${ }^{\circ 14}$ ) désormais absorbée dans le tissu urbain en expansion, le site, après la signature en 1845 d'une ordonnance par Louis-Philippe fixant le dépotoir à La Villette, avait été aménagé au bord d'une darse pour devenir, après 1849, année de sa mise en service, l'unique dépotoir des vidanges parisiennes ${ }^{40}$. L'évolution de la politique d'hygiène et d'assainissement en faveur du tout-à-l'égout allait faire évoluer l'usage du site où seraient construits, à la fin du XIX ${ }^{e}$ siècle, des ateliers d'entretien du matériel de curage des égouts. 


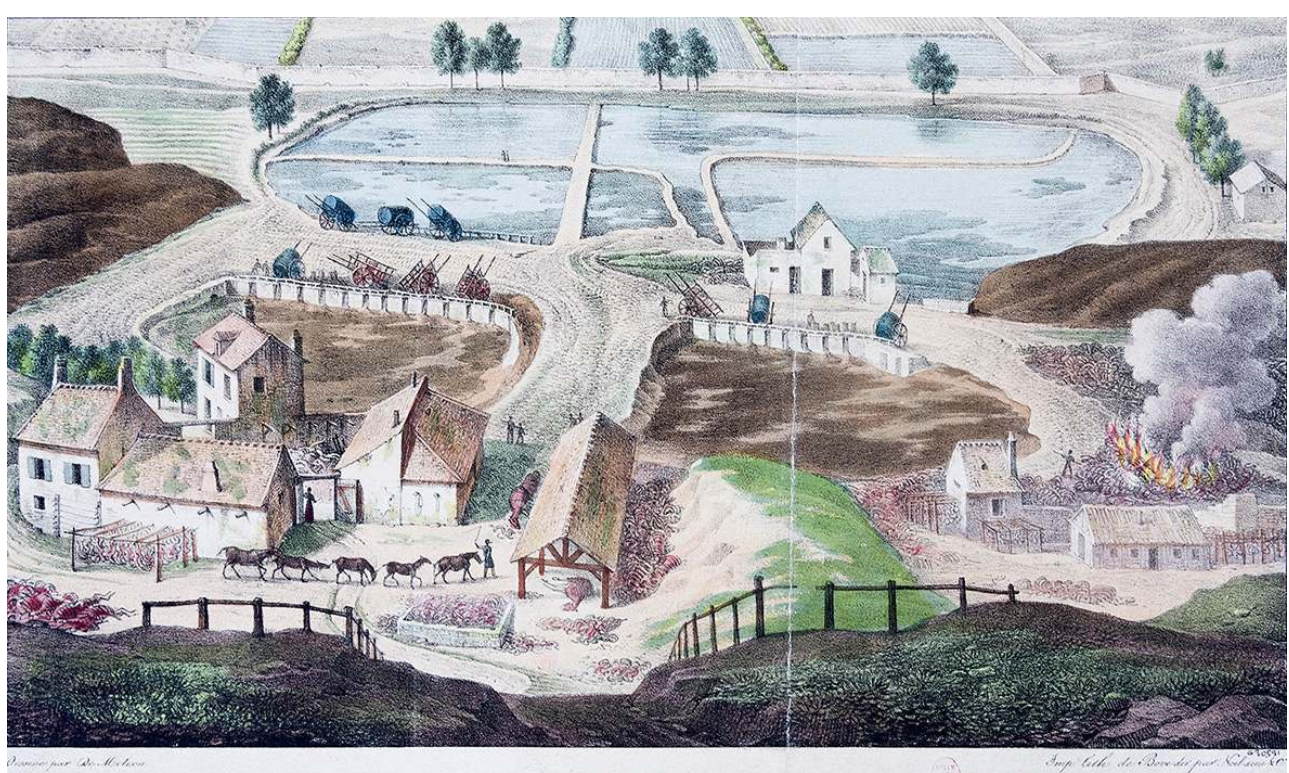

Moléon, Vue du chantier de Mr Chollet, lithographie coloriée, Annales mensuelles de l'Industrie et des Beaux-Arts, pl. 1, (1827), musée Carnavalet, Cabinet des arts graphiques, G.24985.

(c) Mairie de Paris/ Florence Morisson, MPP, 2018.

Dans un bâtiment mitoyen, des salles d'exposition furent aménagées, dont le plan est reproduit en annexe du rapport Ranson, complété à titre comparatif par celui du Musée sanitaire d'Hornsey ${ }^{41}$ en Angleterre. Le rapport du conseiller municipal Georges Lemarchand (1869-1950) de 1908 détaille plus précisément ce plan (fig. $\left.{ }^{\circ} 15\right)$ : trois salles d'exposition, attenantes à une salle d'essais et à un laboratoire, présentaient des appareils hydrauliques, divers types de latrines et d'appareils sanitaires pour les logements de particuliers, ainsi que des modèles réduits ou des appareils de curage des égouts en grandeur nature. 


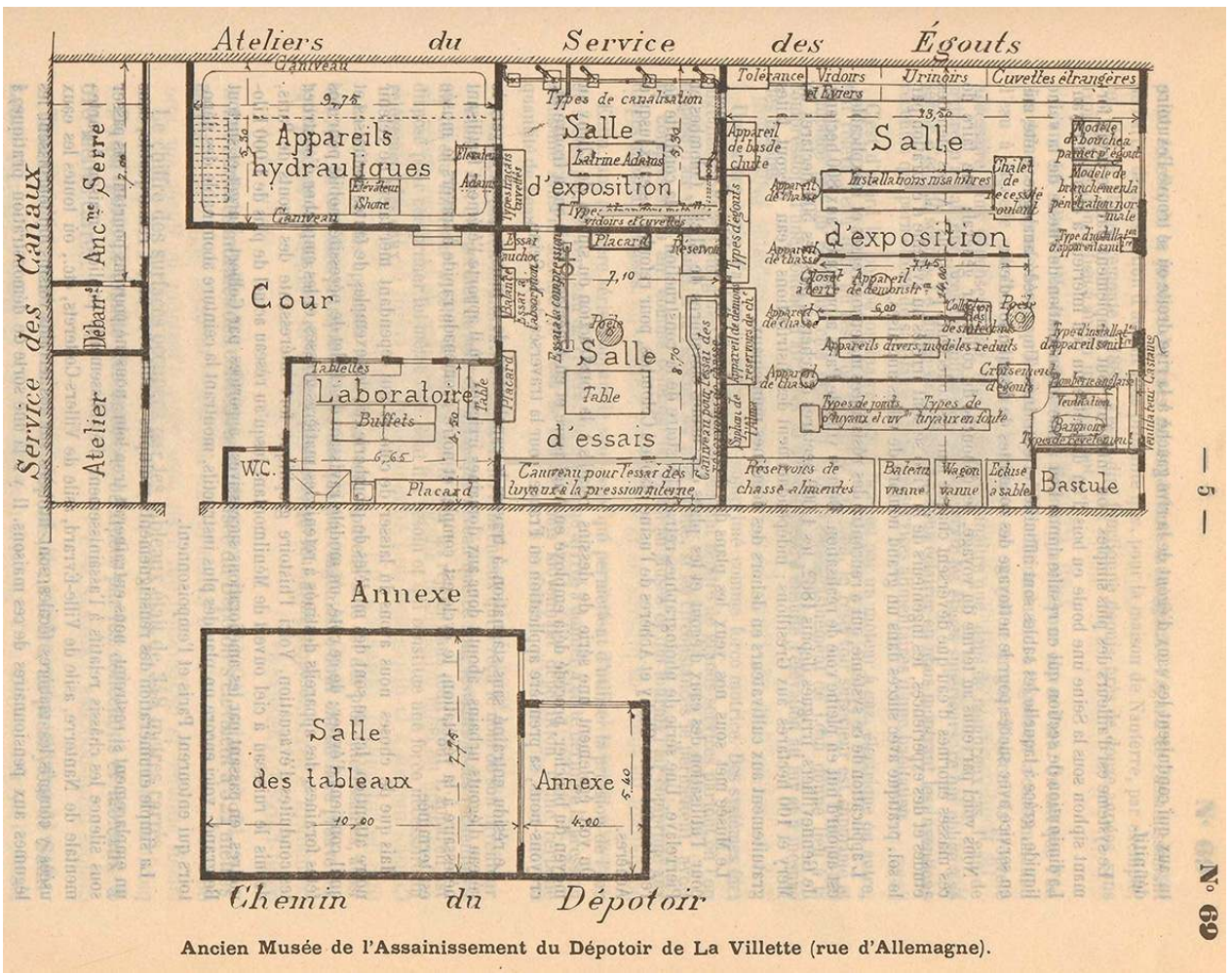

Anonyme, Ancien Musée de l'Assainissement du Dépotoir de La Villette (rue d'Allemagne), Rapport Lemarchand $1908, n^{\circ} 69$

(c) Mairie de Paris/ Florence Morisson, MPP, 2018

À proximité, une annexe contenant une «salle des tableaux » de près de $80 \mathrm{~m}^{2}$, était vraisemblablement destinée à présenter de l'iconographie. Ces divers matériels à vocation pédagogique étaient les reliquats des dispositifs fabriqués pour être présentés dans les Expositions universelles de 1878 et 1889. Cela est corroboré par le rapport de 1911, qui précise : « Il existait en 1908, dans les dépendances du dépotoir de la Villette, une collection d'appareils spéciaux tels que cuvettes de water-closets, modèles d'égouts, tuyauterie sanitaire qui avaient été réunis par le Service des travaux sanitaires dans un but de propagande pour tout ce qui concernait l'installation du tout-à-l'égout». Précédemment, l'Encyclopédie municipale ${ }^{42}$ qualifiait cet ensemble de « Musée sanitaire » :

Un dépôt des collections du Service de l'assainissement ou Musée sanitaire a été installé dans les dépendances du Dépotoir, à La Villette. On y trouve des tableaux, des diagrammes, des modèles et des spécimens d'appareils ayant figuré aux diverses expositions. Un atelier d'essais pour l'expérimentation de nouveaux types d'appareils, cuvettes, réservoirs de chasse, élévateurs, tuyaux en grès, en fonte, etc., et un petit laboratoire pour analyses sommaires, se trouvent adjoints au musée sanitaire. Pour visiter, s'adresser au Service technique de l'assainissement qui délivre des cartes à volonté.

Rappelons que la loi du 10 juillet 1894, qui faisait obligation pour les propriétaires de maisons en bordure de rues pourvues d'un égout public, d'écouler souterrainement à l'égout les matières solides et liquides en provenance des lieux d'aisance et imposait un cabinet d'aisance dans chaque appartement pour toute maison à construire, n'avait pas suffi à vaincre les réticences des propriétaires, qui multiplièrent les procédures juridiques, retardant ainsi l'application du système de l'égout-exutoire complet. En 1900, 
on recensait encore 51500 fosses fixes et, sur 71488 immeubles, seuls 23035 étaient équipés de chutes spéciales pour le tout-à-l'égout ${ }^{43}$. Ce musée avait bien pour vocation de convaincre par la démonstration.

\section{Boulevard de Sébastopol, un véritable musée}

Ces salles furent transférées fin 1911 au 27 boulevard Sébastopol, sous la dénomination de musée municipal d'Hygiène, dit aussi Musée sanitaire (fig. 16).

Figure 16

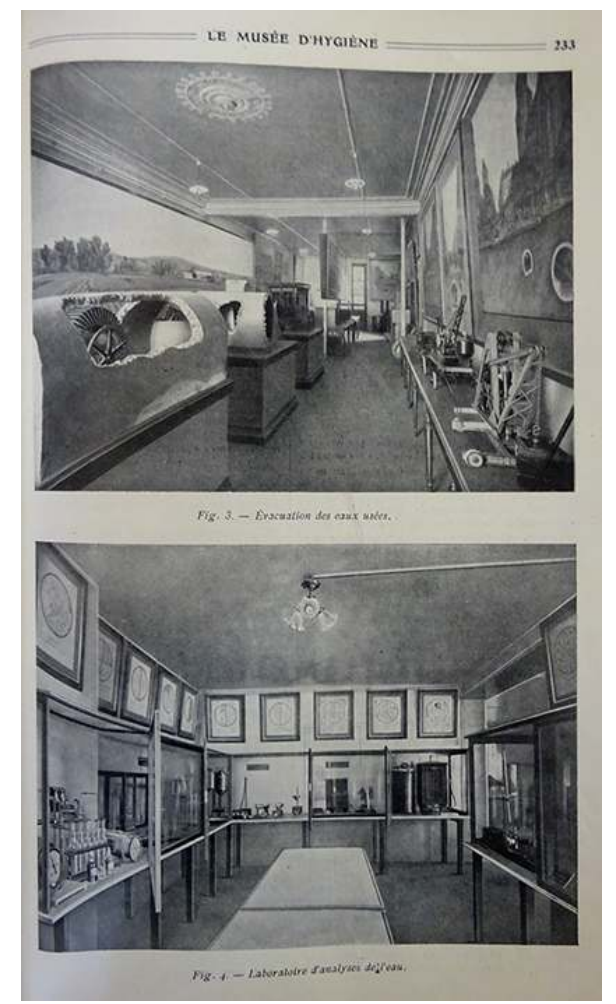

Anonyme, vue de deux salles du nouveau musée d'hygiène, photographies, La Nature, 1912, t. I, p. 233.

(c) Mairie de Paris/ Florence Morisson, MPP, 2018.

Le rapport Lemarchand présenté en novembre 1908 s'avéra décisif, puisqu'il amena au vote de la création et de l'installation de ce musée par la Direction des affaires municipales, dans l'ancien presbytère de l'église Saint-Leu situé entre le boulevard Sébastopol et la rue Saint-Denis ${ }^{44}$ : cette même année, « la vente des terrains du dépotoir obligea l'Administration à déplacer les collections et le Conseil municipal décida la création d'un Musée municipal d'hygiène. L'ancien presbytère [...] vacant depuis la loi de séparation, fut choisi pour y installer le nouveau Musée ». Le projet d'aménagement du bâtiment et de constitution des collections fut confié au chef du bureau administratif des Services d'hygiène, Paul Juillerat (1854-1935), qui en fut le premier conservateur. Au milieu de 1909, Roger Foucault (1852-1929), architecte de l'Administration centrale, commença la transformation du bâtiment pour l'adapter à sa nouvelle destination. Au personnel était adjointe une commission de surveillance et de perfectionnement des Services d'hygiène présidée par le docteur Émile Roux (1853-1933), directeur de l'Institut Pasteur, «chargée de se prononcer sur l'admission des objets offerts ou que 
l'Administration propose d'acquérir ». Le musée comprenait deux corps de bâtiments de trois étages, ouvrant sur le boulevard Sébastopol et sur la rue Saint-Denis, réunis par une salle aménagée en salle de conférences pour 250 à 300 auditeurs. Il comportait, outre le parcours de visite des collections, une bibliothèque (fonds constitués par le don à la Ville de Paris par sa veuve, de l'ancienne bibliothèque de l'ingénieur Durand-Claye, apôtre de l'assainissement de Paris par le tout-à-l'égout), et une salle de travail. Le rapport donne des indications sur la constitution des collections, à laquelle s'était attelée l'administration du musée en avril 1911: «Les différents services qui ont à s'occuper de l'hygiène parisienne ont été invités à se mettre en rapport avec le conservateur pour examiner dans quelle mesure ils pouvaient contribuer à la création des collections. » Une mention spéciale est faite à l'administration générale de l'Assistance publique, qui a d'emblée donné un nombre important d'objets et de documents. Le rapporteur exprime le souhait que "les chefs de tous les services comprennent combien il importe que les services municipaux soient honorablement représentés ». Le conservateur effectua également des démarches auprès des constructeurs, des sociétés, des administrations publiques et privées, et fit exécuter, «sur les crédits assez restreints mis à sa disposition, des pièces anatomiques, des photographies, dessins, etc., que l'on ne trouve pas dans le commerce, et dont la Commission du musée avait autorisé la confection. »

Le rapporteur souligne la «complexité extraordinaire d'une pareille œuvre», loue la présentation de « documents aussi divers, qui mettent à contribution toutes les branches de la science, depuis l'art de l'ingénieur jusqu'à la médecine humaine et vétérinaire ». Il énumère en outre le nom de tous les donateurs privés et publics. La bataille du tout-àl'égout semble gagnée, mais il faut encore de vaincre la résistance des propriétaires qui rechignent à financer son installation dans leurs immeubles et logements. Il s'agit aussi de convertir la population parisienne aux bienfaits de l'hygiène, par une présentation didactique destinée à un public le plus large possible, comprenant les enfants des écoles et les sociétés populaires: le rapporteur loue «l'esprit éminemment pratique qui a présidé aux installations. Tout a été combiné pour servir à la fois à renseigner les techniciens et à instruire le public. Partout où cela a été possible, les appareils ont été installés pour pouvoir être mis en fonctionnement. " Le conservateur a élaboré "un programme organisant des conférences le soir, des visites-conférences pour les écoles, les collectivités de toute nature qui voudront initier leurs membres aux principes de la lutte contre la maladie. [...] Telle qu'elle est réalisée, (cette création) ne sera pas seulement un musée d'hygiène, mais encore [...] une école de l'hygiène ».

Signe de l'importance accordée à cet établissement, l'inauguration par le président du conseil municipal de la première institution de ce genre sur le sol français se déroula, le 6 novembre 1911, en présence du ministre de l'Intérieur et du ministre du Travail et de la Prévoyance sociale, du préfet de la Seine, du préfet de police, de membres du conseil municipal, des directeurs des services, de notabilités scientifiques et "d'hommes intéressés par le progrès social ». Le musée ouvrit au public le lendemain. À compter de cette date et jusqu'au 15 décembre, plus de 3000 personnes en visitèrent les collections.

51 Le rapport d'André Gent (1859-) en décembre 1912 (voir note 37) donne le détail de la répartition des collections en sept sections occupant vingt-huit salles ou galeries. Celle-ci mérite une citation in extenso tant elle reflète cette conception totalisante de l'hygiène dont il était question plus haut, qui recouvre tous les domaines de compétence d'une ville :

$1^{\text {re }}$ section - Hygiène urbaine 
Cette section renferme tout ce qui concerne l'alimentation en eau des villes, l'évacuation des matières usées (vidanges, égouts, champs d'épandage, épuration des eaux usées, etc., etc.) ; l'établissement, l'entretien et le nettoiement de la voie publique ; l'enlèvement et la destruction ou l'utilisation des ordures ménagères ; les plans d'extension des villes; les parcs et squares ; les cimetières; en un mot, tout ce qui contribue à assurer l'hygiène générale et est du ressort des Administrations municipales.

$2^{\mathrm{e}}$ section - Hygiène de l'habitation

Comprend: matériaux de construction, alimentation en eau, évacuation des matières usées, chauffage, ventilation et éclairage; distribution intérieure; mobilier dans ses rapports avec l'hygiène.

$3^{\mathrm{e}}$ section - Prophylaxie des maladies contagieuses

Cette section comporte tout ce qui concerne la désinfection publique ou privée ; l'organisation et le matériel des laboratoires, la bactériologie, la surveillance et le contrôle des eaux d'alimentation; la vaccination, le transport des malades; les appareils à stériliser les objets ou les substances contaminées, etc., etc.

$4^{\mathrm{e}}$ section - Hygiène des collectivités

Application des appareils ou dispositions contenues dans les trois sections précédentes aux habitations collectives: hôpitaux, hospices, lycées, écoles, casernes, prisons, etc.

$5^{\mathrm{e}}$ section - Hygiène alimentaire

Halles et marchés, entrepôts, abattoirs, conservation des denrées alimentaires; surveillance des denrées, répression des fraudes, etc.

$6^{\mathrm{e}}$ section - Hygiène des transports

Chemins de fer, bateaux; voitures publiques ou privées du double point de vue de l'hygiène du voyageur et de la protection de la santé des localités parcourues (évacuation des matières usées notamment).

$7^{\mathrm{e}}$ section - Hygiène sociale

Dans cette section sera placé tout ce qui concerne les habitations à bon marché, la lutte contre la tuberculose et l'alcoolisme; les dispensaires, sanatoriums, refuges ; la protection des enfants, etc., etc.

En même temps, une place y est réservée à tout ce qui touche l'hygiène individuelle, soins de propreté (bains-douches, etc.), exercices physiques, vêtements, etc.

Le musée était ouvert les dimanches, mardis, mercredis et vendredis de midi à 17 heures, sur présentation de cartes pour une visite ou de cartes annuelles délivrées après une demande écrite au préfet de la Seine. Le jeudi était réservé aux visites-conférences organisées pour les écoles et les collectivités. Insistant «sur l'importance qu'elle présentait pour l'éducation morale et sociale de la population ", le préfet a avisé le directeur de l'Enseignement. Le lundi était réservé au nettoyage en grand des salles et des collections, et le samedi était le jour de repos pour le personnel. Des visites-conférences furent organisées le dimanche, notamment pour les sociétés L'art pour tous et les Universités populaires. Le conservateur organisa en 1913 une série de conférences en soirée dans la salle de conférences, auxquelles des spécialistes apportaient leur concours. Début 1903, la bibliothèque spéciale fut ouverte aux travailleurs. Le rapporteur se félicite de l'« empressement de la population et des sociétés d'instruction populaire à profiter des facilités qui leur sont données d'apprendre des notions exactes sur l'hygiène. Cet empressement nous montre bien que la nouvelle institution répond à un besoin actuel ».

Outre le conservateur et son adjoint, « 5 ouvriers spéciaux [étaient] chargés de l'entretien et de la réparation des appareils et du gardiennage du musée ", "un personnel d'élite d'ouvriers spéciaux, choisis avec soin, et auxquels on doit la construction de la plupart des meubles, tables, vitrines, etc., qui étaient nécessaires, dans des conditions de prix de revient auxquelles nous ne sommes pas habitués ». 

organisationnelles du travail, se fait jour un enjeu mémoriel et transmissif par le biais de la patrimonialisation, dans la décision de créer une mission sur le patrimoine professionnel municipal. Prenant en considération, en se fondant sur une approche pluridisciplinaire, des métiers en évolution ou voués à disparaitre, ce souci se fonde sur une prise de conscience de la valeur technique, culturelle, sociale et économique de ce patrimoine, qu'il soit matériel ou immatériel, en tentant d'articuler ces deux dimensions par un indispensable travail de documentation contextualisante qui seul, par la connaissance, permet de détacher leur conservation de sa seule portée nostalgique au profit d'un ancrage dynamique dans le présent. Vecteur de fierté professionnelle et de prise de conscience citoyenne du fonctionnement de la ville, notre objectif est d'en renverser la perception passéiste pour en faire une ressource commune permettant de comprendre comment se fait la ville, associant pédagogie et demande sociale et culturelle, disponible pour les agents municipaux et les Parisiens.

interne destinée au personnel municipal, quelques vitrines isolées et des présentations plus importantes ont conservé quelques ensembles, assurant de manière souterraine une continuité remarquable dans les préoccupations de l'édilité et de l'administration parisiennes : souci pédagogique de formation interne et d'éducation des Parisiens.

Au cours des visites de repérage, nous avons entendu parler à plusieurs reprises d'un projet, envisagé dans les années 1990, de musée de la fonction publique parisienne, destiné à mettre en valeur le travail des agents municipaux. Nous ne proposons pas de créer ce qui serait un dispositif lourd, mais de mettre en œuvre une structure qui sécuriserait les collections d'objets en les conservant pour partie dans leurs sites actuels (espaces mémoriels qui seraient des "micro-musées »), et pour partie dans une réserve visitable (accueillant les objets patrimoniaux que les services ne peuvent ou ne veulent conserver), qui pourrait devenir un centre de ressources à destination des directions de l'administration parisienne et des agents, et le point d'ancrage d'un tourisme destiné aux Parisiens de toutes générations.

Musée-forum plutôt que musée-temple, faisant écho aux développements les plus actuels de la muséologie pratiquée par nombre de musées de société, ce dispositif permettrait de répondre à tous les objectifs assignés. Il assurerait la conservation matérielle des objets à valeur historique (inventaire du patrimoine professionnel municipal, marquage des objets et veille, étude et documentation, présentation dans des dispositifs sécurisés), en conférant à ceux-ci une meilleure visibilité (in situ et dans une réserve visitable, tête de 
pont du dispositif) tout en permettant leur étude et leur valorisation - voire, à terme, la poursuite de l'usage de certains d'entre eux par une organisation de dépôts similaire à celle du Mobilier national ou du Fonds municipal d'art contemporain. Il devrait sécuriser celle des archives audiovisuelles (récits de métier, de carrière, filmages de gestes et savoir-faire, visites commentées filmées, clips de communication interne, réalisés par nos soins ou par des auteurs externes), pour leur donner une diffusion dans le futur par le truchement d'une base de données qui pourrait être enrichie par les acteurs des activités recensées.

\section{NOTES}

1. - Je remercie Christine Oger pour sa lecture vigilante et ses conseils avisés.

2. - Depuis la loi du 31 décembre 1975, l'élection d'un maire a induit d'importantes transformations.

3. - Relayées par une participation à des foires, notamment la Foire de Paris, fondée en 1904 par la chambre de commerce et d'industrie de Paris et un certain nombre d'organisations professionnelles.

4. - BRUNEL, Georges. «La formation des musées de la Ville de Paris et le développement de l'administration des Beaux-Arts ». Dans GEORGEL, Chantal (dir.). Collections électroniques de l'INHA, Choisir Paris: les grandes donations aux musées de la Ville de Paris, voir le site: https:// inha.revues.org/6884 [consulté le 08/11/2018].

5. - Ces magasins n'étaient pas visitables. À notre connaissance, aucune trace d'inventaire n'en subsiste.

6. - Incorporée à la Direction des travaux de Paris à la fin de l'Empire, dirigée pendant 20 ans par Jean-Charles Alphand (1817-1891), elle sera par la suite de nouveau rattachée directement au préfet. En 1903 est créé un Service unique des beaux-arts et des musées.

7. - MARTIN, Léon (dir.). Encyclopédie municipale de la Ville de Paris. Paris: Neger-Reeb, 1902, p. 1307-1308.

8. - BRUNEL, Georges. Art. cit. En 1897, séparation d'avec la Bibliothèque historique pour laquelle fut acquis l'hôtel Le Peletier de Saint-Fargeau.

9. - Bâtiment construit avenue du Président-Wilson aux frais de la duchesse de Galliera afin d'abriter les collections qu'elle destinait à la Ville par legs, legs auquel elle renonça en raison de l'évolution de la situation politique en France, au profit de la Ville de Gênes, en 1888. Après la Seconde Guerre mondiale, les collections artistiques municipales furent réunies avec celles du Petit Palais.

10. - Celle-ci allait être transférée en 1962 dans l'ancien hôtel de Sens, qui avait échappé de peu à la démolition à la fin du XIX ${ }^{\mathrm{e}}$ et au début $\mathrm{du} \mathrm{Xx}^{\mathrm{e}}$ siècles grâce à l'action conjuguée de la Société des amis des monuments parisiens et de la Commission du Vieux Paris, et à son acquisition par la Ville en 1911.

11. - MARTIN, Léon. Op. cit., p. 713. Distinct du musée dépendant du ministère de l'Instruction publique, situé 41 rue Gay-Lussac, $5^{\mathrm{e}}$ arrondissement.

12. - Conseil municipal, 1896, $\mathrm{n}^{\circ} 182$, Rapport présenté par M. A. Ranson, au nom de la $6^{e}$ souscommission du Budget et du Contrôle sur les chapitres IV, articles 16 (Location d'un local pour l'Inspection générale de l'assainissement) et VIII (Assainissement de l'habitation), p. 6-7. 
13. - MARTIN, Léon. Op. cit., p. 1185.

14. - BERU, François de. "Quand les rues étaient pavées en bois : l'usine municipale de Javel ». Bulletin de la Société d'histoire et d'architecture du $15^{\circ}$ arrondissement de Paris, printemps 2016, $\mathrm{n}^{\circ} 47$, p. 8. Cette usine se situait à l'angle du quai de Javel et de la rue des Cévennes.

15. - Consécutive à la concession le 25 janvier 1856 de l'éclairage au gaz de Paris à la Compagnie parisienne du gaz. D'abord installé quai de Béthune, le laboratoire fut transféré en 1874 dans l'annexe est de l'Hôtel de Ville, rue Lobau. Le regroupement des laboratoires se fit à la faveur d'une installation $10 \mathrm{bd}$ Kellermann, où ils prirent le nom de Laboratoire des équipements de rue (activités éclairage et circulation).

16. - Conseil municipal de Paris, $1877, \mathrm{n}^{\circ} 2$, Rapport présenté par M. Viollet-Le-Duc, au nom de la $5^{e}$ Commission, concernant la participation des services municipaux à l'Exposition universelle de 1878. Paris : Imprimerie municipale, Hôtel de Ville, 1877 - $5^{\mathrm{e}}$ Commission.

17. - LANDAU, Bernard. «La fabrication des rues de Paris au XIX ${ }^{e}$ siècle : l'enfer vaincu et l'utopie dépassée ». Les Annales de la recherche urbaine, 1992, n 57-58, "Espaces publics en ville », voir le site : http://www.persee.fr/doc/aru_0180-930x_1992_num_57_1_1696 [consulté le 08/11/2018].

18. - RASMUSSEN, Anne. "L'hygiène en congrès (1852-1912), circulation et configurations internationales ». Dans BOURDELAIS, Patrice (dir.). Les Hygiénistes: enjeux, modèles et pratiques ( XVIII ${ }^{e}$-XX ${ }^{e}$ siècles). Paris : Belin, 2001, p. 213-239.

19. - Comptes rendus sténographiques des congrès internationaux de l'Exposition universelle de 1878. $\mathrm{N}^{\mathrm{O}} 10$ de la série : Congrès international d'hygiène, tenu à Paris du $1^{\mathrm{er}}$ au 10 août 1878.

20. - En 1882, institution par le préfet d'une commission technique de l'assainissement de Paris, rassemblant 40 spécialistes, où s'affrontent des médecins favorables à la canalisation, et des ingénieurs partisans du tout-à-l'égout. À l'issue des délibérations, le rapport général préconise l'adoption du tout-à-l'égout. Le conseil municipal adopte en avril 1884 un projet de règlement soumis à une enquête publique dans les 20 arrondissements ; JACQUEMET, Gérard. "Urbanisme parisien: la bataille du tout-à-l'égout à la fin $\mathrm{du} \mathrm{XIX}^{\mathrm{e}}$ siècle». Revue d'histoire moderne et contemporaine, octobre-décembre 1979, t. XXVI; GOUBERT, Jean-Pierre. La Conquête de l'eau, l'avènement de la santé à l'âge industriel. Paris : R. Laffont, 1986.

21. - DU CAMP, Maxime. Paris, ses organes, ses fonctions et sa vie dans la seconde moitié du XIXe siècle. Paris : Hachette, 1869-1875.

22. - MARTIN, Léon. Op. cit., 1903, p. 1518.

23. - Coupure de presse conservée au Cabinet des arts graphiques du musée Carnavalet, fonds Mœurs 16/3 17, signée G.B., datant sans doute de 1865 (G.36113).

24. - LAROULANDIE, Fabrice. "Les égouts de Paris au XIX siècle: l'enfer vaincu et l'utopie dépassée ». Dans BONZON, Thierry, BOUCHERON, Patrick, CHALEARD, Jean-Louis, et al. Idées de villes, villes idéales. Fontenay/Saint-Cloud : ENS, « Les Cahiers de Fontenay », 1993, p. 107-140.

25. - MARTIN, Léon. Op. cit., p. 1518.

26. - Archives de Paris, Registre de la Visite publique des égouts, VO3 179, 1903-1926.

27. - En vue d'une réouverture fin 2019, une rénovation est engagée par la Ville de Paris sur un programme architectural et muséographique confié à l'agence Frenak et Jullien.

28. - Conseil municipal de Paris, 1877, $\mathrm{n}^{\circ} 2$, Rapport présenté par M. Viollet-Le-Duc ... Op. cit.

29. - « Les eaux et les égouts de Paris. À l'Exposition universelle de 1878 ». Revue scientifique de la France et de l'étranger, 5 octobre 1878, mis en ligne par Denis Blaizot sur le site: http:// sciences.gloubik.info/spip.php?article1362 [consulté le 13/11/2018].

30. - Catalogue de l'Exposition spéciale de la Ville de Paris et du Département de la Seine. Paris : Impr Chaix, 1889, voir le site: http://www.worldfairs.info/expopavillondetails.php? expo_id=6\&pavillon [consulté le 13/11/2018].

31. - Conseil municipal de Paris, Rapport de M. de Bouteiller, au nom de la Commission municipale de l'Exposition, sur l'exposition spéciale de la Ville en 1889, 30 novembre 1887, nº 139. 
32. - Exposition universelle de Paris en 1900. Catalogue de l'Exposition spéciale de la Ville de Paris et du Département de la Seine. Paris : Imprimerie Chaix, 1900.

33. - Dirigé par Georges Bechmann (1848-1927), ingénieur en chef des Ponts et Chaussées.

34. - Cat. 717 : roue Meunier en service à l'usine Mazas, 1 modèle; cat. 722 : bateau-vanne en service dans un collecteur, 1 modèle; cat. 723 : wagon-vanne en service dans un collecteur, 1 modèle ; cat. 724 : réservoirs de chasse avec appareil à 1 départ en service sur égout, 1 modèle ; cat. 725 : réservoirs de chasse avec appareil à 2 départs en service sur égout, 1 modèle ; cat. 726 : bassin à sable avec drague mobile sur rails, 1 modèle; cat. $727:$ drague sur rails, suspendue à la voûte de la galerie, 1 modèle ; cat. 729 : grue à vapeur montée sur chariot attelé. Funiculaire pour le transport des seaux, 1 modèle ; cat. 730 : tracteur électrique sur trolleys, avec wagons pour le transport des sables, 1 modèle. Plusieurs de ces modèles réduits sont présentés dans des vitrines de l'actuelle Visite publique des égouts.

35. - Situé au rez-de-chaussée de l'aile nord du palais de Tokyo, désormais musée d'Art moderne de la Ville de Paris.

36. - Sur la thématique de l'insalubrité, au musée Carnavalet, deux maquettes du Studio E.P.O.C. représentent deux secteurs de la Zone. Le dossier d'œuvre de la maquette PM.82 mentionne: «Établie pour le Service des expositions du Service d'architecture et d'urbanisme de la Ville de Paris / Février 1950 - Dépôt du Service de topographie et d'urbanisme de la Préfecture de la Seine / Exp. : Exp. Univ. 1937, Pavillon de la Ville de Paris ».

37. - Conseil municipal de Paris, 1896, $\mathrm{n}^{\circ} 182$, Rapport présenté par M. Ranson, au nom de la $6^{e}$ souscommission du Budget et du Contrôle des chapitres IV et VIII. Paris : Imprimerie municipale, Hôtel de Ville ; 1898, Rapport Fortin ; 1900, Rapport Barillier, Rapport Galli ; 1901, Rapport Bussat ; 1902, Rapport Paris ; 1903, Rapport Larrige ; 1905, Rapport Massard ; 1906, Rapport Jolibois ; 1908, Rapport Ambroise Rendu, Rapport Lemarchand ; 1911, $\mathrm{n}^{\circ} 138$ : Rapport Charles Guillard, Rapport au nom de la $6^{e}$ Commission, sur le budget du Musée d'hygiène, présenté par M. Charles Guillard, Conseiller municipal, 29 décembre $1911 ; 1912, \mathrm{n}^{\circ} 135$, Rapport André Gent, Rapport au nom de la $6^{e}$ Commission sur le budget du Musée d'hygiène, présenté par M. André Gent, Conseiller municipal, 21 décembre 1912.

38. - Rebaptisée avenue Jean-Jaurès en 1914.

39. - Terme ici compris dans le sens de l'enlèvement quotidien des ordures dans les villes.

40. - Sur place, celles-ci étaient déchargées nuitamment des tombereaux acheminés par la rue d'Allemagne, dans des citernes aménagées dans un hangar. Elles y étaient séparées entre les parties solides, transportées en tonneaux sur des péniches rejoignant la voirie de Bondy aménagée dans la forêt éponyme en 1817 - et les parties liquides envoyées par une conduite forcée longeant le canal Saint-Denis, jusqu'à la même destination. À Bondy, chacun des deux types d'effluent était mis à décanter dans de vastes bassins, puis retraité en poudrette agricole et en produits industriels dérivés de l'ammoniaque.

41. - «Highgate Museum of sanitary appliances ", ouvert en 1892 par le conseil municipal de Hornsey (faubourg de Londres) dans un dépôt de charbon, fermé en 1928. Voir le site : Hornsey, including Highgate: Social and cultural activities | British... [consulté le 13/11/2018]. Voir aussi : LONG, Helen C. The Edwardian House. The middle-class Home in Britain, 1888-1914. Manchester/ New York : Manchester University Press, 1993, p. 57.

42. - MARTIN, Léon. Op. cit., p. 1086.

43. - LAROULANDIE, Fabrice. Art. cit., p. 136.

44. - Conseil municipal de Paris, $1911, \mathrm{n}^{\circ} 138$ : Rapport Charles Guillard. Op. cit.

45. - Le Conseil municipal - Nos édiles, Paris, Publications du Journal Municipal La Cité, éditions de 1926 et 1933.

46. - « Deux musées parisiens dispersés 1- le Musée d'hygiène de la Ville de Paris - 2- le Musée des travaux publics ». Le Vieux Paris, mars $1971,17^{\mathrm{e}}$ année, $\mathrm{n}^{\circ}$ 68, p. 1-4. 


\section{RÉSUMÉS}

Désireuse de mettre en valeur et de faire connaître l'excellence technologique et la capacité d'innovation de ses services sous la Troisième République, époque de grande modernisation de Paris qui s'inscrit dans le prolongement du Second Empire, l'administration de la capitale a présenté son activité dans le cadre de diverses Expositions universelles, et ouvert dans leur sillage des «musées professionnels ", avec des objectifs de formation interne et de promotion de son action, en matière notamment d'hygiène et de santé publique, ces sites étant ouverts au public. Après un bref tableau de la situation actuelle, cet article évoque les premiers "musées » techniques municipaux. Puis un focus sur la présentation des efforts de la Ville de Paris en matière d'hygiène et d'assainissement approfondit la genèse administrative du musée municipal de l'Hygiène, ancêtre de l'actuelle visite publique des égouts.

In order to draw attention to its technical accomplishments and its capacities for innovation, the Parisian municipal administration presented its achievements at several universal exhibitions during the Third Republic. This was a period of considerable modernisation of the city, in pursuance of the work begun under Haussmann during the Second Empire. Following these exhibitions, the city opened 'professional museums', with the aim of promoting its initiatives in fields such as hygiene and public health and providing training for its employees. These places were open to the public. This article offers a survey of the present-day situation in Paris and examines the first technical museums created in the city. Its analysis of the efforts undertaken here by the city administration give us a better understanding of how the municipal museum of hygiene came into existence within the administration. This museum is the ancestor of the visits organised today to the Paris sewer system.

\section{INDEX}

Mots-clés : hygiène, musée de l'Hygiène, musée sanitaire, musée technique, musée industriel, éducation à l'hygiène, Expositions universelles, égouts, visite des égouts, Ville de Paris, Eugène Viollet-le-Duc

Keywords : hygiene, hygiene museum, sanitary museum, technical museum, industrial museum, hygiene education, universal exhibitions, seweres, visits to the sewers, city of Paris Eugène Viollet-le-Duc

\section{AUTEUR}

\section{MIRIAM SIMON}

Conservatrice générale, responsable de la Mission patrimoine professionnel, Ville de Paris miriam.simon@paris.fr 